NISTIR 8215

\title{
Forensic Latent Fingerprint Preprocessing Assessment
}

\author{
Paul Lee \\ Haiying Guan \\ Andrew Dienstfrey \\ Mary Theofanos \\ Brian Stanton \\ Matthew T. Schwarz
}

This publication is available free of charge from:

https://doi.org/10.6028/NIST.IR.8215

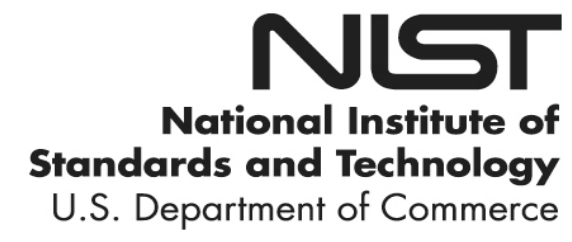


NISTIR 8215

\section{Forensic Latent Fingerprint Preprocessing Assessment}

Paul Lee

Visualization and Usability Group Information Technology Laboratory

Haiying Guan

Multimodal Information Group

Information Technology Laboratory

Andrew Dienstfrey Mathematical Analysis and Modeling Group Information Technology Laboratory

Mary Theofanos Office of Data and Informatics Material Measurements Laboratory

Brian Stanton Visualization and Usability Group Information Technology Laboratory

Matthew T. Schwarz Schwarz Forensic Enterprises

This publication is available free of charge from: https://doi.org/10.6028/NIST.IR.8215

June 2018

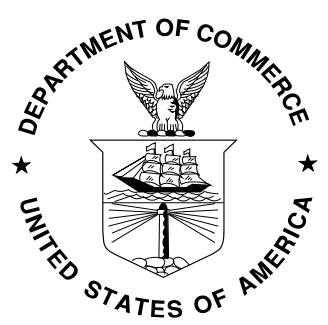

U.S. Department of Commerce Wilbur L. Ross, Jr., Secretary

National Institute of Standards and Technology Walter Copan, NIST Director and Undersecretary of Commerce for Standards and Technology 


\section{TABLE OF CONTENTS}

$1 \quad$ INTRODUCTION $\quad 1$

1.1 PURPOSE AND SCOPE

1.2 LATENT FINGERPRINTS 3

1.3 FINGERPRINT PREPROCESSING

1.4 REPORT STRUCTURE 6

2 STUDY DESIGN $\quad 7$

2.1 OBJECTIVE

2.2 INITIAL DATASET $\quad 7$

2.3 EXPERIMENTAL DESIGN

2.3.1 Examiner Duties 9

2.3.2 EFS Details 9

2.3.3 Image Distribution $\quad 10$

2.3.4 Flat/Rolled Fingerprint Collection $\quad 10$

3 DATABASES 11

3.1 FINGERPRINT RECORDS DATABASE 12

3.1.1 FILENAMES AND NAMING SCHEME

3.1.2 RECORD NUMBERING SCHEME

3.1.3 LATENT IMAGES 16

3.1.4 PREPROCESSED IMAGES 17

3.1.5 FLAT/ROLLED IMAGES 18

3.1.6 BENCHMARK FILES AND ALIGNMENT 19

3.1.7 MARKUP FILES

3.1.8 MARKUP METADATA FILES

3.1.9 MASKS 24

3.1.10 MATCHED LATENT AND PROCESSED 25

3.1.11 METADATA FILES 26

4 MEASUREMENTS 28

4.1 QUALITY MAP SCALE 28

4.2 GBU LATENT VALUES 31

4.3 INTERSECTION SET CALCULATION 33

5 QUALITY METRICS 33

$5.1 \quad$ MINUTIAE GAIN PERCENTAGE

5.2 LATENT VALUE DETERMINATION

5.3 QUALITY CONFIDENCE SCORE 38

6 DISCUSSION AND FUTURE WORK $\quad 40$

7 ACKNOWLEDGEMENTS $\quad 41$

8 DISCLAIMER $\quad 41$

9 REFERENCES $\quad 42$ 


\section{Executive Summary:}

Latent fingerprints are made visible from the surface of objects using a variety of methods including physical and chemical processing, adapted illumination sources, and photographs. Unfortunately, the prints collected directly from a crime scene or from physical evidence may be incomplete or damaged, to the point of being considered unsuitable to send directly to latent fingerprint examiners for identification or to Automated Fingerprint Identification System (AFIS) for recognition. As a result, most latent fingerprint images must be preprocessed to enhance fingerprint information, at the same time suppressing interference arising from noise and otherwise unwanted image features. This preprocessing phase occurs after the latent print is collected from the crime scene and digitalized by a scanner or camera, and before the image analysis and process phase such as minutiae markup or latent print identification. The preprocessing results can be extraordinary, transforming raw images with little or no value into ones suitable for evidentiary analysis. Despite the importance of this step, there exist few databases designed especially for the scientific study of latent fingerprint image preprocessing, and even fewer standards to guide this workflow.

To address this research gap, we conducted a study in which a group of trained Latent Print Examiners provided Extended Feature Set (EFS) markups of a series of latent images. This report provides a brief introduction into fingerprint preprocessing, discussion regarding the experimental design, structure, and contents of the latent fingerprint image database, and details of proposed preprocessing efficacy metrics. Additionally, we present preliminary analysis of these metrics when applied to images in our database.

Previously Guan et al. [6] presented the performance metrics and analysis of the metrics as applied to a 'union' minutiae set. In this report, we comprehensively document the contents of the records database including the latent images, the EFS markup files, metadata, markup logs, and region of interest masks. Additionally, we present the results of the performance metrics as applied to the intersection minutiae set of EFS markup data.

\section{Keywords:}

Forensics; latent fingerprint; preprocessing; quality metrics; minutiae 


\section{INTRODUCTION}

\subsection{PURPOSE AND SCOPE}

The complete process chain of latent fingerprint analysis includes lifting, preprocessing, minutiae extraction or markup, matching for identification/recognition as shown in Figure 1 (Figure 1.1 in [1]). For many fields dealing with latent images, people use the phrase "processing" to deal with all image related procedure. However, in the latent fingerprint examination, the step after the latent image digitalization, and before the actual minutiae analysis and extraction, is called "preprocessing" as shown between the blocks labeled '40' and ' 50 ' in Figure 1. Some academia research community also call this step "enhancement” [2] [3] [4] [5]. In the report, we use phrase "preprocessing” to differentiate it from image processing that focuses on the actual minutiae detection and extraction. Some key components of the workflow shown in Figure 1 are well studied in current literature, and are implemented in existing fingerprint analysis systems. For example, years of research are embodied in software tools for automatic fingerprint feature extraction, fingerprint matching, and the identification of flat and/or rolled fingerprint images. However, the latent fingerprint preprocessing step is comparatively overlooked in current research. This is problematic as preprocessing is the one of the initial steps of the analysis process. Prints resulting from preprocessing are critical to subsequent analysis, and poorly preprocessed images may greatly reduce a feature extraction algorithms' performance. In turn, poor feature extraction reduces the number of data points available for matching and eventually leads to detrimental consequences for reproducibility, traceability, and quantification of accuracy.

To address this problem, we focus on this underexplored link of latent fingerprint preprocessing comparison and analysis. The ultimate goal of our research is to develop a rigorous, evidence-based foundation for image preprocessing in the forensic analysis workflow. Instead of directly working on preprocessing algorithms, we start to build the foundation for the future study of latent preprocessing, which includes but is not limited to preprocessing database design and collection, and studies comparing before/after images and their marked-up features to deeply understand the effect of preprocessing in real-world forensic applications. 


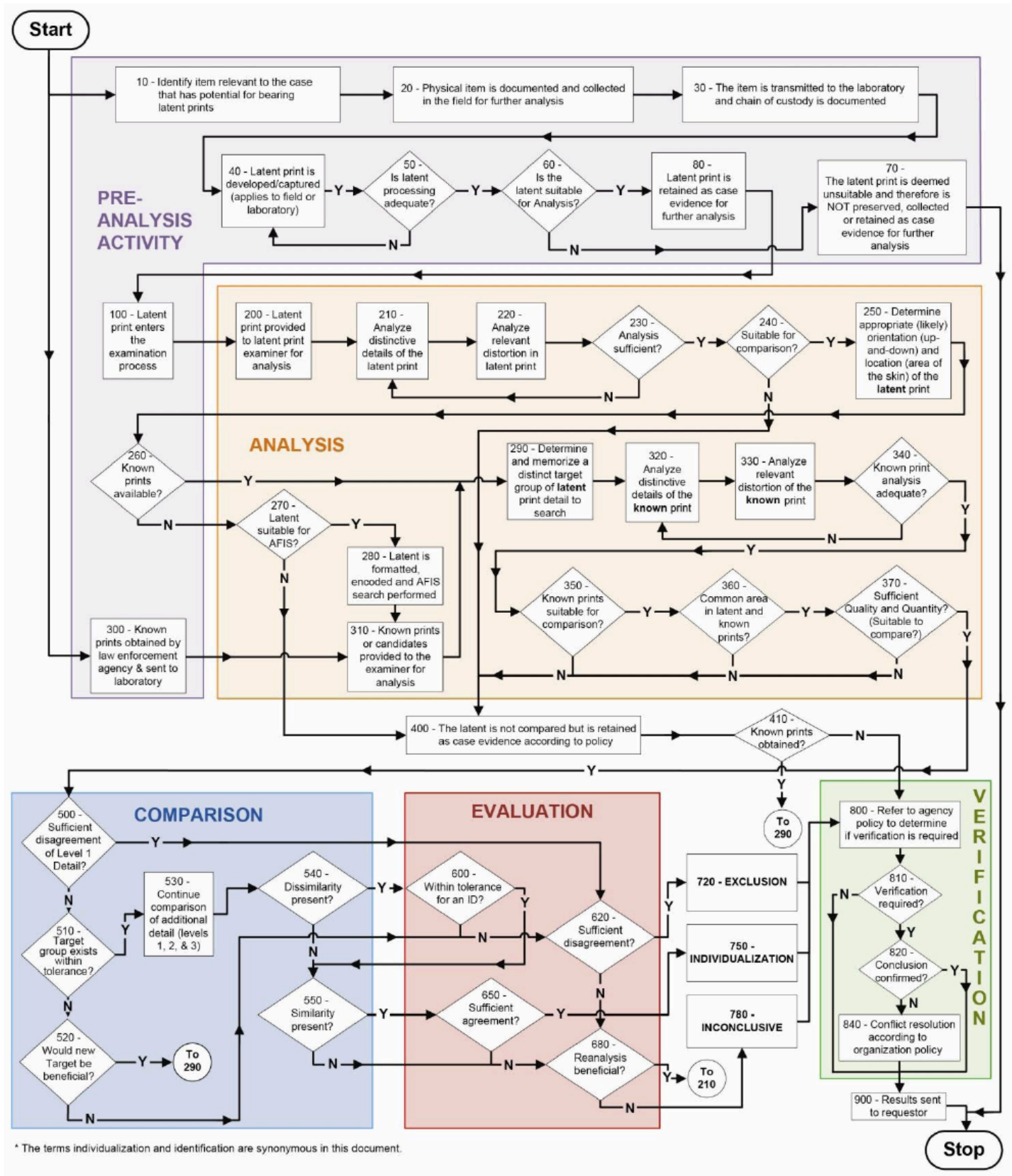

Figure 1: The Latent Print Examination Process Map (Figure 1.1 in [1]) 
The contributions of the report are three-fold: first, we have created a fingerprint preprocessing dataset $^{1}$ with EFS markup files, latent-card fingerprint pairs, and metadata. Second, we propose a scientific research methodology from experimental design to comparison analysis. Finally, we perform a quantitative analysis of the ability to identify fingerprint minutiae comparing latent fingerprint images before and after preprocessing. The results of our analysis provide the early foundations for a systematic and scientific basis for latent fingerprint preprocessing analysis, as well as serve as test case for the development of comparable analysis for other image-based methods in forensic science.

Our work will facilitate the creation of a quantitative and reproducible analysis to support a scientific basis for preprocessing applied to latent fingerprint images. While the complete uncertainty analysis for the fingerprint matching problem is beyond scope, the targeted research investigating latent fingerprint preprocessing will play a non-trivial role in complete analysis of fingerprint identification sensitivity, selectivity, and uncertainty to be performed in future. Transition and field deployment of our experiment results will be greatly accelerated by close collaborations with subject matter experts participating in our dataset collection and generation.

\subsection{LATENT FINGERPRINTS}

Latent fingerprints are friction ridge impressions left unintentionally on various surfaces. Images of latent fingerprints can be obtained (i.e., "lifted” or "developed") using numerous methods, ranging from precision photography to complex physical and chemical processing techniques [2]. These images play a critical role in forensic science and are routinely used as evidence in criminal cases. Unfortunately, due to the complexity of image collection and the imperfect nature of latent impressions, the fingerprint information contained in the images collected directly from a crime scene may be incomplete or hard to identify. This can lead to latent fingerprint images of dubious quality.

Consider the latent fingerprint examples shown in Figure 2. In each example, the full details of the impression are obscured, whether it be due to background textures, colors, patterns, and/or interference from prints deposited by other fingers. These, along with poor quality of ridge impressions, a limited fingerprint area, and large nonlinear distortion due to pressure variations are the major challenges for latent fingerprint analysis [3][4][5][8][9]. Due to the low signal quality of the fingerprint image in relation to other systematic image features, the initial fingerprint image quality may only be of marginal value for identification. In some extreme cases, potentially usable prints are classified as having 'no value', a designation that

1 The latent fingerprint images are from a training data set provided by the course from FORAY technologies and Schwarz Forensic Enterprises, Inc. 
indicates that the image is unsuitable for feature markup, comparison, entry into an identity database, or for use in conjunction with fingerprint identification software.

To mitigate this issue, current practice allows for Latent Print Examiners (LPE) to perform image preprocessing prior to feature markup and identification analysis. The forensics community currently uses a variety of image editing and preprocessing tools to improve image quality, with the goal of enhancing local level features called minutiae. Minutiae, along with other ridge characteristics, are the determining factors behind the uniqueness of each fingerprint [7].

The results of preprocessing on the images in Figure 2 can be seen in Figure 3, with A2, B2, $\mathrm{C} 2$, and D2 corresponding to A1, B1, C1, and D1 respectively. As is shown, preprocessing can transform latent images with little or no value ${ }^{2}$ into ones suitable for evidentiary analysis.

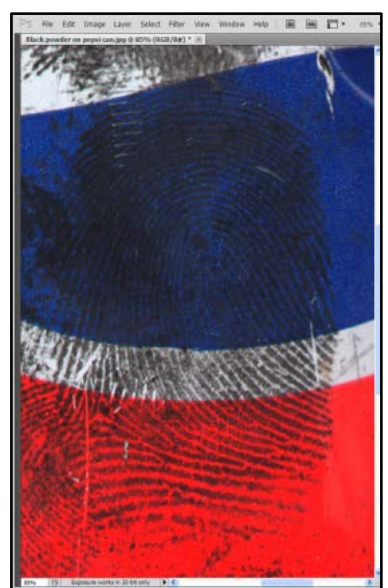

A1

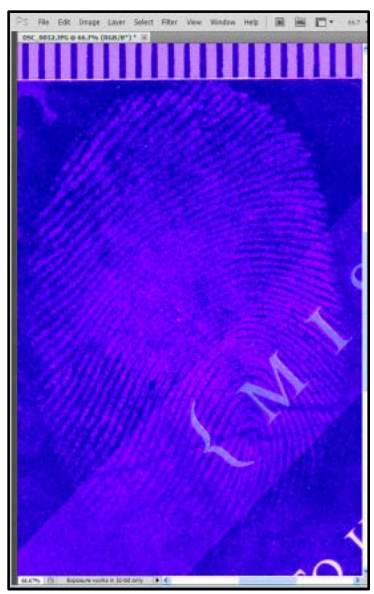

B1

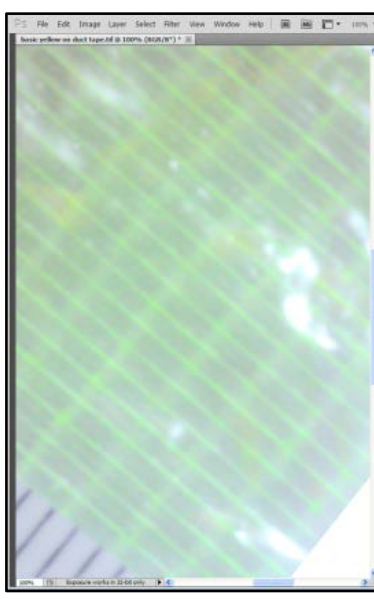

C1

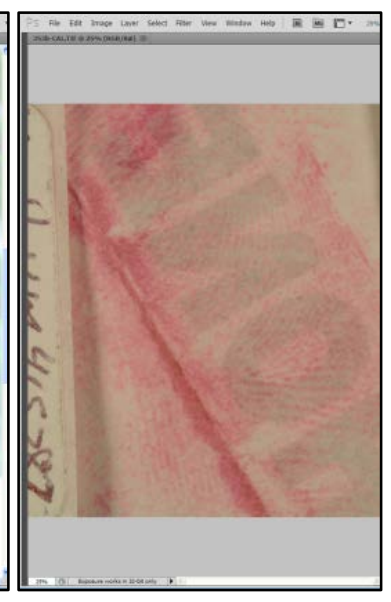

D1

Figure 2: Examples of Initial Latent Fingerprint Images

2 “A “no value” determination preemptively states that no individualization or exclusion determination could be made using the impression, regardless of quality of the comparison prints" [11]. 


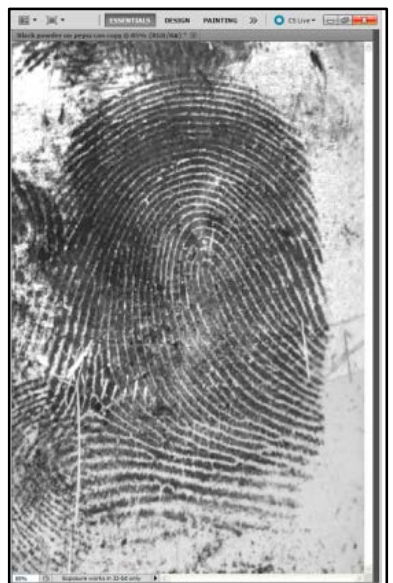

A2

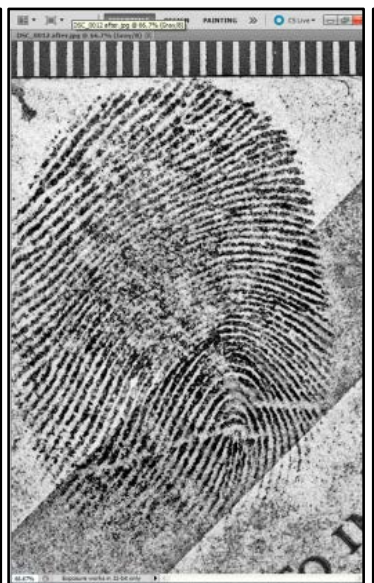

B2

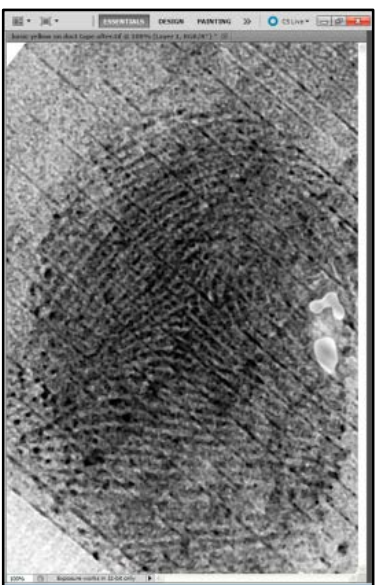

$\mathrm{C} 2$

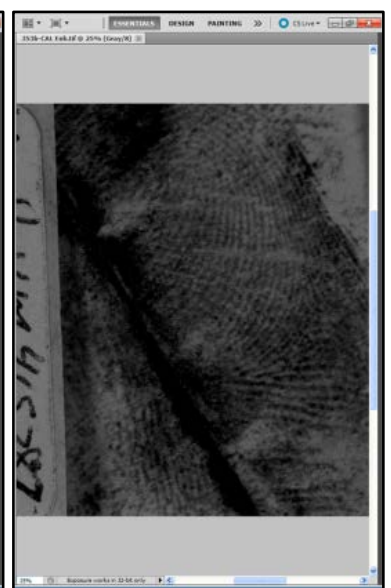

D2

Figure 3: Examples of Preprocessed Latent Fingerprint Images

\subsection{FINGERPRINT PREPROCESSING}

Preprocessing is a complex collection of activities by which latent print examiners improve the retrievable information in a latent image while avoiding any edits that alter critical aspects of this information. For example, judicious use of Adobe Photoshop ${ }^{3}$ has dodge tool and burn tool. "The Dodge tool and the Burn tool lighten or darken areas of the image. These tools are based on a traditional darkroom technique for regulating exposure on specific areas of a print. Photographers hold back light to lighten an area on the print (dodging) or increase the exposure to darken areas on a print (burning). The more you paint over an area with the Dodge or Burn tool, the lighter or darker it becomes." 4 burn tool results in higher contrast ridge details, but improper use or overuse may lead to accidentally darkened valleys that blend together with nearby ridges, or adding false minutiae or obscuring potentially usable minutiae. This need for discretion can lead to preprocessing completion times that can vary from a few minutes to half an hour per latent impression [10]. Regardless of the latent image's initial quality, LPEs follow a general preprocessing workflow, which can be broken down into three tasks: Address Color, Evaluate Contrast and Clarity, and Evaluate Noise [11][12]. The first task deals with examining the color information of an image and evaluating if that information obscures minutiae data. If so, a LPE has several options available, including:

- Editing single color channels to suppress colored backgrounds.

\footnotetext{
3 See Section 8 for NIST Disclaimer

4 https:/helpx.adobe.com/photoshop/using/dodge-burn-image-areas.html
} 
- Changing the saturation of the image to affect color intensity.

- Adjusting color brightness at the pixel level with respect to a color channel.

After issues with color are addressed, the LPE moves onto the adjusting the contrast and clarity of various minutiae. Options available to the LPE include (but are not limited to):

- Using the Dodge and Burn tools to lighten valleys and darken ridges.

- Modifying the shadows and highlights of either entire images or small, localized regions, with precise tone details controllable via individual sliders.

- Redistributing contrast by using curves, which selectively stretch or compress input tones.

With the image's color corrected and the contrast adjusted to boost information visibility, the final step is to address any miscellaneous noise, which can include individual pixels, small areas of white, and background patterns. Techniques used for this step include:

- Blurring minor background noise by using the Dust and Scratches tool.

- Sharpening the image using the Unsharp Mask feature if the image is blurry or has a soft focus.

- Using custom plugins that remove consistent patterns, such as horizontal lines on a piece of paper.

Due to the variable nature of latent impressions, the number and type of court acceptable techniques used by an LPE vary from image to image. However, the overall goal for and result of these three steps is the same: a preprocessed latent image that improves the minutiae information that can be retrieved, which can lead to faster and more accurate identification.

\subsection{REPORT STRUCTURE}

The remainder of this report is divided into the following major sections:

Section 2 provides an overview of the study's objectives, details regarding the database of initial latent fingerprint images used as the base for our study, and an outline of the study's experimental design. For the latter, we include notes on how fingerprints were distributed to the various examiners, the three phases of markup, ground truth markup data, and the tools used by the latent print examiners.

Section 3 covers the structure of the newly generated database. The three types of files contained within each record - images, markup data, and image metadata - are defined and explored, with examples of each. 
Section 4 defines the set of algorithms used to quantify image quality. Processes and formulas for quality map measurement, minutiae crossover, and intersection minutiae set calculation are covered here.

Section 5 contains our analysis results, covering the three proposed quality measurement metrics - latent value determination and re-categorization, minutiae percentage gain, and quality confidence score changes. Each of these metrics is explored by defining what aspect of quality it measures, what impact that aspect has on the forensic process and the results of our analysis.

Section 6 summarizes this report's overall observations and conclusions, along with details on our future work with this database and in fingerprint preprocessing workspace.

\section{STUDY DESIGN}

\subsection{OBJECTIVE}

The chief goal of our study was to determine whether and to what extent latent fingerprint preprocessing improves the ability to gain information in the identification of latent impressions, as well as to what extent it transforms latent images with no comparison value into images that can be used for analysis. We focused on quantifiable changes in three criteria: detectable fingerprint minutiae, latent value determinations, and quality confidence score changes. Each of these metrics are defined and explored within Section 5. Changes in preserved feature information between latent images presented in color vs. grayscale were also examined, to gauge if the color information within an image has any measurable effect on fingerprint information content.

Unlike previous studies examining preprocessing [11][12], our study does not use examiner results as a point for analysis. Markups among examiners are not compared to each other, nor is examiner performance evaluated. Additionally, no identifying information is kept or linked to any of the latent images.

\subsection{INITIAL DATASET}

The latent fingerprint images used for examiner markup were sourced from a previously created database that isolated several steps in the preprocessing workflow. This database consists of 89 latent fingerprint records, each containing latent image pairs and a latent examiner action log. The collection process began with unprocessed latent images that were developed using a cross-section of physical and chemical lifting techniques including ninhydrin, silver magnesium powder, white powder, bi-chromatic powder, bi-chromatic mag powder, and black ink. The types of surfaces from which they were lifted varied, which led to 
ridges, valleys, and other minutiae being obscured by differing colors, textures, and pressure levels. Naturally, this resulted in a wide range of initial impression quality.

The lifted prints were then scanned using high-resolution flatbed scanners and then given to latent print examiners to undergo preprocessing, following the workflow outlined in section 1.3. All preprocessing was done using Adobe Photoshop, the commonly used image analysis tool by practicing latent print examiners today. As the LPEs processed the images, each of their actions were automatically recorded and exported to an external text document as per existing best-practice guidelines [13], resulting in an action log of each image transformation.

Examples of recorded actions include:

- $\quad$ Cropping 5 , burning, and dodging 6

- Inverting colors for color correction ${ }^{7}$

- Parameters used for shadow manipulation ${ }^{8}$

- Changing individual color channels ${ }^{9}$

- Grayscale conversion ${ }^{10}$

- Applying filters for pattern removal ${ }^{11}$

- Editing brightness and contrast levels ${ }^{12}$

In addition to individual editing actions, file open/close timestamps and file paths were also noted. The resulting file triplets (the latent fingerprint image, the preprocessed image, and the action log) for each fingerprint record has proven to be an invaluable source of controlled data for developing scientific analyses of forensic image preprocessing. We intentionally used this initial dataset as the basis for the next step in our research - creating an information rich

\footnotetext{
5 https://helpx.adobe.com/photoshop/using/crop-straighten-photos.html

6 https:/helpx.adobe.com/photoshop/using/dodge-burn-image-areas.html

7 https://helpx.adobe.com/photoshop-elements/using/adjustment-filters.html

8 https://helpx.adobe.com/photoshop/using/adjust-shadow-highlight-detail.html

9 https://helpx.adobe.com/photoshop/using/color-monochrome-adjustments-using-channels.html

10 https://helpx.adobe.com/photoshop/using/converting-color-modes.html

11 https://helpx.adobe.com/photoshop/using/applying-smart-filters.html

12 https://helpx.adobe.com/photoshop/using/apply-brightness-contrast-adjustment.html
} 
database of latent fingerprint images, fingerprint card images, minutiae markup files, quality map data, and fingerprint metadata.

\subsection{EXPERIMENTAL DESIGN}

The updated version of the latent preprocessing database contains 89 fingerprint records. Structurally, each record contains:

- Five latent fingerprint images, varying in resolution, color presence, and preprocessed status.

- The EFS markups for these five images, saved in the Latent Friction Features Search format (.lffs), as defined by the Electronic Biometric Transmission Specification [14].

- The source finger's card image and it's EFS markup.

- Metadata files containing key information regarding the LPE ID, the source finger ID, value determinations, image resolutions, the method used to lift the print, etc.

- A log of actions taken by the examiner while preprocessing the latent image.

\subsubsection{Examiner Duties}

The EFS markups for the latent images were performed by a team of 9 independent LPEs. Each LPE was given a series of latent images, spread across three phases, and were tasked with marking the various features within the EFS.

\subsubsection{EFS Details}

The EFS was developed by Noblis ${ }^{13}$ (http://www.noblis.org) in collaboration with the Federal Bureau of Investigations and standardizes the diverse fingerprint image metadata considered useful for identification analysis. The EFS augments the ridge-flow information contained within a fingerprint image by inserting standardized indications of features including: ridge quality maps, incipient ridges, minutiae, cores, deltas, and others. LPEs followed instructions using the ACEV (Analysis, Comparison, Evaluation, and Verification) methodology to assess images for the presence of friction ridges, fingerprint information, and the confidence of such information. Examiners performed all markup analysis using the Universal Latent Workstation (ULW), version 6 or newer [15]. Enhancement tools present in the ULW Latent Editor software or in any other software that the examiner might have available to them were strictly forbidden.

Under our study, three versions of each latent image were marked by examiners: the original latent image in color ('BeforePreprocess-Color'), the original latent image in grayscale

13 See Section 8 for NIST Disclaimer 
('BeforePreprocess-GrayScale'), and the preprocessed After image ('AfterPreprocessGrayScale’).

\subsubsection{Image Distribution}

Images were distributed and presented to the LPE's in a predetermined order per the following assignment criteria:

- Examiners received and marked one image at a time.

- No information regarding whether or not the image has undergone pre-processing was given.

- The 'before' image (the original image without any preprocessing) and its 'after' image (its preprocessed image) are assigned to different examiners in most cases.

- Each examiner received at least one Good quality image and one Bad quality image. The remainder were a mix of Good, Bad, and Ugly quality images [16]

- Finger source distribution was randomized, ensuring approximately the same distribution amongst examiners.

- Each latent image was marked by two different LPEs.

Images were distributed to the LPEs in three phases. In the first phase, examiners were only given unprocessed latent images converted to grayscale and asked to mark features as normal. In the second phase, examiners were given the corresponding unprocessed latent images as they were originally scanned, with all color and pattern interference preserved. The examiners were asked to mark up the color latent image, but were allowed to reference their markup in phase one. This was done to explore the possibility of feature information being lost during grayscale conversion. In the final phase, examiners were given 'AfterPreprocess-GrayScale' images that did not correspond to any unprocessed latent image marked previously, and were instructed to mark features as normal. No references were given in this phase, unlike the previous phase.

\subsubsection{Flat/Rolled Fingerprint Collection}

In addition to the latent image markups, we collected the corresponding flat/rolled print for each record. Each LPE, separate from the examiners who took part in the markup process in 2.3.3, was tasked with the markup of these images using the same EFS categories. This markup acts as 'ground truth' EFS data, which serves as a basis of comparison between 'Before' and 'After' images. The established feature information baseline that can be derived from the ground truth data allows us to set an expectation of how much feature information can be extracted from an ideal print.

Using these figures, the improvement can be compared from preprocessing to the total amount of information contained within a fingerprint, to determine if it results in an appreciable 
difference. Without the ground truth context, a fifty percent improvement in detected minutiae may seem fantastic, but this improvement may not result in any notable performance gain improvement when the preprocessed image is put through an identification algorithm. However, the markup ground truth data is only part of the complete picture.

To accurately capture the extent of improved information capture, images were generated displaying the latent impression overlaid on top of the card flat/rolled image were generated. These serve as 'benchmark' images and are useful for both quickly visualizing latent and card flat/rolled markup data, and allowing researchers and LPEs to easily compare minutiae sources. By examining the positioning of a marked minutiae in the latent image and comparing it to nearby marked minutiae in the card flat/rolled image, an examiner can confirm minutiae correspondence. Accurate minutiae correspondence is key, as many of the ground truth markup data contain significant minutiae clusters. Distinguishing between a vague similarity in a general area and being able to pinpoint exactly which minutiae could be detected allows for a much more comprehensive determination of to what areas preprocessing was able to improve the most. This data can be further categorized into features that were extracted from the 'Before' latent image and features that only became visible in the 'After' image.

Before the comparison images could be generated however, the rotational ground truth data is required to be calculated. Without knowing the exact rotational translation needed to align the two prints, accurately overlaying the images is a very challenging research topic. In current forensics practice, this image registration process is accomplished as a sub-task of EFS feature comparison, which leads to varied methodology and results. In the present study, we sought to eliminate this source of variability. For each latent record, an independent examiner identified a number $(>3)$ of benchmark minutiae that could be found on both the latent image and its associated card flat/rolled image. Corresponding minutiae were indicated by different colors, and were ideally separated as much as possible throughout the region of interest. A color point detection algorithm identified the locations of corresponding features, and a least-squares algorithm was used to estimate the rigid transformation parameters (rotation and translation) that would transform the benchmark latent orientation to that of the benchmark flat/rolled print.

\section{DATABASES}

This section reviews the structure and contents of the fingerprint records database, which contains the latent images, EFS markup data, metadata, and benchmarks.

Each record in the records database consists of a directory containing 29 files. For organizational purposes we consider these files as belonging to one of eight categories. The definition and details of each are discussed in the sub-sections below, along with a list of files and example images. 


\subsection{FINGERPRINT RECORDS DATABASE}

The first of the two databases is the fingerprint records database. The current database contains 89 fingerprint records, stored as folders. Each folder contains a variety of files pertaining to a captured print, it's markup, and other relevant information.

\subsubsection{FILENAMES AND NAMING SCHEME}

Files are named by first including 'study' and the record number, and then the relevant file type name. For example, the unprocessed latent image at its original resolution for record 001 would be named 'study001-latent.tiff'. This naming scheme continues for all files found within that record. Files for record 002 would start with 'study002' and so forth, with specific file type names remaining consistent. Example filenames noted below will follow the 'study\#filename.extension' format.

Table 1: Records Database Filenames

\begin{tabular}{|c|c|}
\hline \multicolumn{2}{|l|}{ Records Database Filenames } \\
\hline Latent Images & Description \\
\hline study\#-latent.tiff & $\begin{array}{l}\text { The original, unprocessed 'Before' latent image, with colors } \\
\text { and resolution preserved. }\end{array}$ \\
\hline study\#-latent-gray-1000ppi.tiff & $\begin{array}{l}\text { The unprocessed latent image, 'BeforePreprocess- } \\
\text { GrayScale', is grayscale image with resolution scaled to } \\
1000 \text { ppi for use with the ULW Latent Editor Software. }\end{array}$ \\
\hline study\#-latent-color-1000ppi.tiff & $\begin{array}{l}\text { The unprocessed latent image, 'BeforePreprocess-Color', is } \\
\text { color preserved image with resolution scaled to } 1000 \text { ppi for } \\
\text { use with the ULW Latent Editor Software. }\end{array}$ \\
\hline \multicolumn{2}{|l|}{ Preprocessed Images } \\
\hline study\#-processed.tiff & $\begin{array}{l}\text { The processed latent image, 'AfterPreprocess-GrayScale', is } \\
\text { the image with original resolution after various } \\
\text { preprocessing procedures have been applied, including } \\
\text { cropping, tinting, grayscale conversion, and burning. Exact } \\
\text { actions taken are recorded in the meta.docx log file. }\end{array}$ \\
\hline study\#-processed-1000ppi.tiff & $\begin{array}{l}\text { The processed latent image, 'AfterPreprocess-GrayScale', is } \\
\text { the image with } 1000 \text { ppi resolution for use with the ULW } \\
\text { Latent Editor Software. }\end{array}$ \\
\hline
\end{tabular}




\begin{tabular}{|c|c|}
\hline \multicolumn{2}{|l|}{ Benchmark Images } \\
\hline study\#-benchmark-latent.tiff & $\begin{array}{l}\text { The processed latent image, 'AfterPreprocess', with color } \\
\text { dot labels on several minutiae and grayscale elsewhere, is } \\
\text { used as a benchmark for rotational alignment. Contains } \\
\text { colored minutiae positions that are scanned and matched to } \\
\text { the relevant minutiae in the benchmark card image. }\end{array}$ \\
\hline study\#-benchmark-source.tiff & $\begin{array}{l}\text { The card flat/rolled image used as a benchmark for rotational } \\
\text { alignment. Obtained from a fingerprint card file as would be } \\
\text { collected by the Federal Bureau of Investigation (FBI). } \\
\text { Contains specific colored minutiae locations that are } \\
\text { scanned and matched to the colored minutiae in the } \\
\text { benchmark latent image. }\end{array}$ \\
\hline \multicolumn{2}{|l|}{ Flat/Rolled Images } \\
\hline study\#-source.tiff & $\begin{array}{l}\text { The unaltered card flat/rolled image. Obtained from a FBI } \\
\text { fingerprint card file. Used in rotational alignment and } \\
\text { minutiae markup for ground truth acquisition. In general, } \\
\text { much higher quality (clarity, background noise, colors, etc.) } \\
\text { than the latent fingerprint image. }\end{array}$ \\
\hline \multicolumn{2}{|l|}{ Latent Markup Files } \\
\hline study\#-efs-latent-gray-1.lffs & $\begin{array}{l}\text { The first of two latent markup files generated by latent print } \\
\text { examiners during the 'BeforePreprocess-GrayScale ' round } \\
\text { of examiner markup. Based off the } 1000 \text { ppi grayscale } \\
\text { version of the latent image. }\end{array}$ \\
\hline study\#-efs-latent-gray-2.lffs & $\begin{array}{l}\text { The second of the two latent markup files generated by latent } \\
\text { print examiners during the 'BeforePreprocess-GrayScale ' } \\
\text { round of examiner markup. Based off the } 1000 \text { ppi grayscale } \\
\text { version of the latent image. }\end{array}$ \\
\hline study\#-efs-latent-color-1.lffs & $\begin{array}{l}\text { The first of two latent markup files generated by latent print } \\
\text { examiners during the 'BeforePreprocess-Color' round of } \\
\text { examiner markup. Based off the } 1000 \text { ppi color version of } \\
\text { the latent image. Markup was performed by the examiner } \\
\text { assigned to the first color latent markup. }\end{array}$ \\
\hline study\#-efs-latent-color-2.lffs & $\begin{array}{l}\text { The second of the two latent markup files generated by latent } \\
\text { print examiners during the 'BeforePreprocess-Color' round } \\
\text { of examiner markup. Based off the } 1000 \text { ppi color version of }\end{array}$ \\
\hline
\end{tabular}




\begin{tabular}{|c|c|}
\hline & $\begin{array}{l}\text { the latent image. Markup was performed by the examiner } \\
\text { assigned to the second color latent markup. }\end{array}$ \\
\hline study\#-efs-processed-1.lffs & $\begin{array}{l}\text { The first of two latent markup files generated by latent print } \\
\text { examiners during the 'AfterPreprocess-GrayScale' round of } \\
\text { examiner markup. Based off the } 1000 \text { ppi grayscale version } \\
\text { of the preprocessed image. Markup was performed by an } \\
\text { examiner not assigned to previous markup rounds for the } \\
\text { record. }\end{array}$ \\
\hline study\#-efs-processed-2.lffs & $\begin{array}{l}\text { The second of the two latent markup files generated by latent } \\
\text { print examiners during the 'AfterPreprocess-GrayScale' } \\
\text { round of examiner markup. Based off the } 1000 \text { ppi grayscale } \\
\text { version of the latent image. Markup was performed by an } \\
\text { examiner not assigned to previous markup rounds for the } \\
\text { record. }\end{array}$ \\
\hline study\#-efs-source.lffs & $\begin{array}{l}\text { The markup file generated from the card flat/rolled } \\
\text { fingerprint image. Represents the ground truth for feature } \\
\text { information contained in a fingerprint. Markup was } \\
\text { performed by an examiner uninvolved in the latent markup } \\
\text { phases. }\end{array}$ \\
\hline \multicolumn{2}{|c|}{ Derived - Latent Markup Logs } \\
\hline study\#-efs-latent-color-1.txt & $\begin{array}{l}\text { The metadata file corresponding to the first of two } \\
\text { 'BeforePreprocess-Color' latent markup files. All metadata } \\
\text { files contain information about transactions, image } \\
\text { properties, feature data, and the ridge quality map. }\end{array}$ \\
\hline study\#-efs-latent-color-2.txt & $\begin{array}{l}\text { The metadata file corresponding to the second of two } \\
\text { 'BeforePreprocess-Color' latent markup files. }\end{array}$ \\
\hline study\#-efs-latent-gray-1.txt & $\begin{array}{l}\text { The metadata file corresponding to the first of two } \\
\text { 'BeforePreprocess-GrayScale ' latent markup files. }\end{array}$ \\
\hline study\#-efs-latent-gray-2.txt & $\begin{array}{l}\text { The metadata file corresponding to the second of two } \\
\text { 'BeforePreprocess-GrayScale ' latent markup files. }\end{array}$ \\
\hline study\#-efs-processed-1.txt & $\begin{array}{l}\text { The metadata file corresponding to the first of two } \\
\text { 'AfterPreprocess-GrayScale' latent markup files. }\end{array}$ \\
\hline study\#-efs-processed-2.txt & $\begin{array}{l}\text { The metadata file corresponding to the second of two } \\
\text { 'AfterPreprocess-GrayScale' latent markup files. }\end{array}$ \\
\hline
\end{tabular}




\begin{tabular}{|l|l|}
\hline study\#-efs-source.txt & $\begin{array}{l}\text { The metadata file corresponding to the card flat/rolled } \\
\text { markup file. }\end{array}$ \\
\hline Derived - Latent Markup Masks & $\begin{array}{l}\text { The composite latent region of interest from the two } \\
\text { BeforePreprocess-Color EFS markup files, used to calculate } \\
\text { the ellipse and its diameter. }\end{array}$ \\
\hline study\#-color-mask.png & $\begin{array}{l}\text { The composite latent region of interest from the two } \\
\text { BeforePreprocess-GrayScale EFS markup files, used to } \\
\text { calculate the ellipse and its diameter. }\end{array}$ \\
\hline study\#-processed-mask.png & $\begin{array}{l}\text { The composite latent region of interest from the two } \\
\text { AfterPreprocess-GrayScale' EFS markup files, used to } \\
\text { calculate the ellipse and its diameter. }\end{array}$ \\
\hline Metadata Files & \multicolumn{1}{|l|}{} \\
\hline study\#-info.csv & $\begin{array}{l}\text { Miscellaneous information about the record, including the } \\
\text { source finger, acquisition type, GBU value determination, } \\
\text { the scanner ID, examiner IDs, and the image resolutions. }\end{array}$ \\
\hline study\#-poly.txt & $\begin{array}{l}\text { A log of examiner actions performed during the } \\
\text { preprocessing procedure. }\end{array}$ \\
\hline study\#-meta.docx & $\begin{array}{l}\text { A list of pixels defining the rectangular region of interest for } \\
\text { the latent image containing usable fingerprint information. } \\
\text { fingerprint mask. }\end{array}$ \\
\hline study-roi.dat & $\begin{array}{l}\text { The transformation matrix used to register a latent } \\
\text { fingerprint image to its corresponding card flat/rolled image. }\end{array}$ \\
\hline
\end{tabular}

\subsubsection{RECORD NUMBERING SCHEME}

Each of the records were numbered according to the type of fingerprint that it contained. The different types include independent latent images, chief scanner duplications, remaining enhancements, child images, parent images, unusable images, and misaligned images. Each of the different types and their classification parameters are listed below. 
0-99: Independent Latent Images (54) - Unique, latent fingerprints unrelated to any other record in the database. Parent images, child images, and images with multiple enhancements were sorted into the categories below.

101-199: Enhancement Duplications (24) - Latent images that were independently preprocessed multiple times on separate occasions.

201-299: Scanner Duplications (11) - Latent fingerprint images scanned more than once if examiners believed that the scanner directly influenced the quality of the image.

301-350: Child Images (27) - Cropped images containing only one fingerprint, sourced from multi-print, parent images. These records do not contain the original latent image, as it would contain the other fingerprints present in the parent image. Instead, the -latent-color-1000ppi.tiff and -latent-gray-1000ppi.tiff images are created by isolating and excising the individual fingerprints from the parent latent image.

351-399: Parent Images (12) - A latent image containing multiple fingerprints. Each fingerprint contained in the parent image is cropped and separated into a child image. Markup is only performed for the individual child images; no markup is performed on the parent image.

401-499: No-value Images (9) - Images were deemed as 'no-value’ for a variety of reasons including incomplete fingerprints, image resolution differences, and images of exceedingly poor quality.

501-599: Rotated Images (2) - Latent images that were rotated during the enhancement process. While the enhancements still produce a valid preprocessed image, the varied rotation is not compatible with our alignment research. To keep the contents of the Independent Latent Images category consistent, we classify these images separately.

Due to the child fingerprint images lacking the initial latent image, parent fingerprint images missing markup data, and the quality issues present for the unusable and misaligned categories, these images were not included in the final version of the records database.

\subsubsection{LATENT IMAGES}

Latent images are images of fingerprint impressions that have been lifted off various objects, including documents, magazines, furniture, etc. These original or 'Before' images are the basis for all markup and comparison, and are generally of much lower quality than the same finger's card flat/rolled fingerprint image. Preprocessing techniques aim to turn low quality latent fingerprint images into images much easier for an AFIS to identify.

There are three latent images in each fingerprint record. The first is the latent image as it was originally scanned. All color information and resolution settings are unchanged. The second is 
the latent image set to a resolution of $1000 \mathrm{ppi}$. The resolution change was necessary as the ULW Latent Editor software used for markup requires 1000 ppi resolution. The third image is the same 1000 ppi latent image but this time converted in grayscale, which is the standard color scheme used during preprocessing. We make the distinction between color and grayscale images to test if there is minutiae information available in the colored images that cannot be seen in the grayscale versions. If there is significantly more information gleaned from the images when the examiners can view both, there may be merit in giving them access to both in future forensic examinations. The file type names for these images are 'study\#-latent.tiff, 'study\#-latent-color-1000ppi.tiff', and 'study\#-latent-gray-1000ppi.tiff' respectively. Examples of each type of image can be seen in Figure 4.

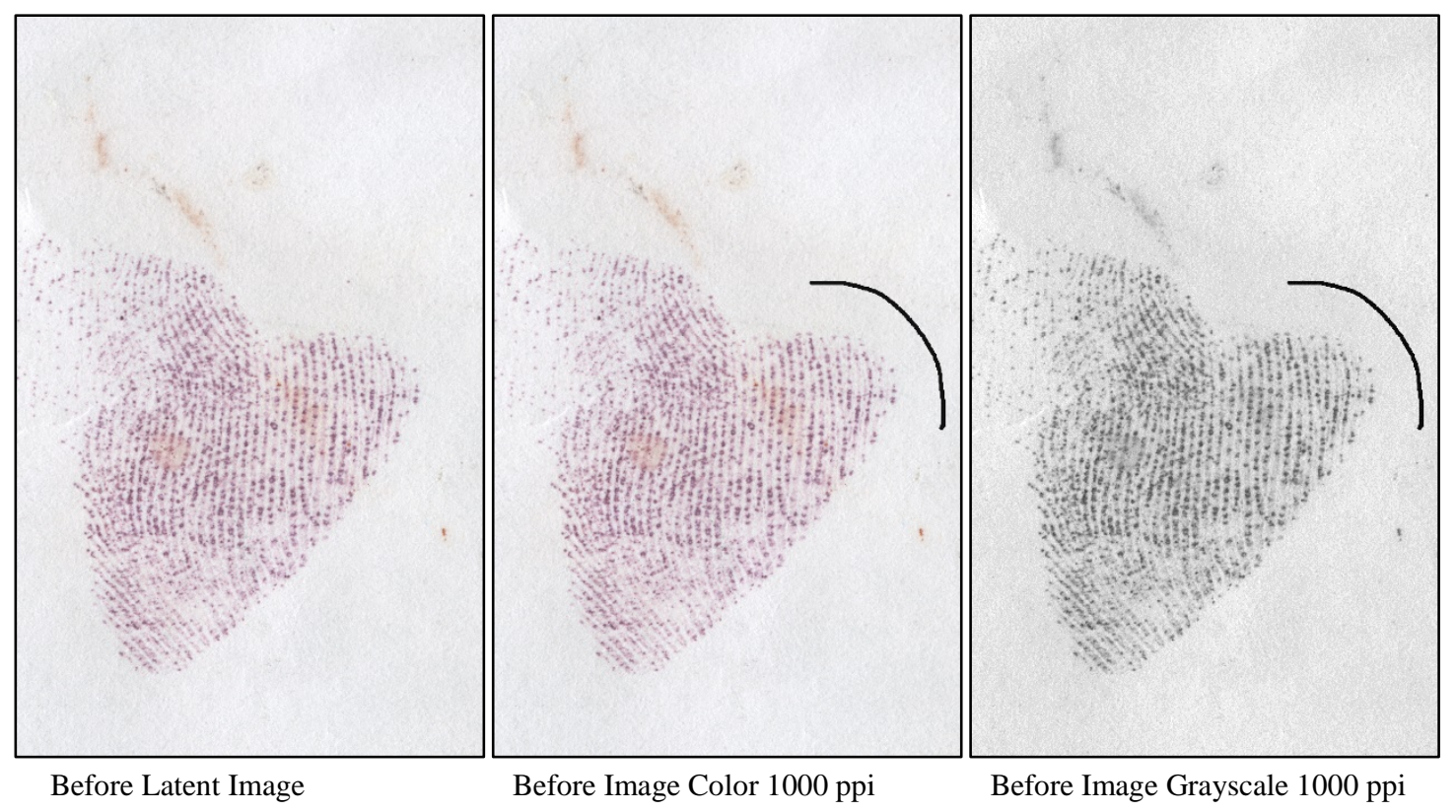

Figure 4: Record 001 Latent Before Image Set

\subsubsection{PREPROCESSED IMAGES}

Preprocessed or 'After' images are the latent fingerprint images after they have undergone enhancement by a latent print examiner. The aim of fingerprint preprocessing is to generate images that contain more feature information than the unprocessed or 'Before' latent image. For most cases, this holds true - the improved image clarity reveals clearer ridge endings, bifurcations, and other types of minutia, which leads to a faster and more accurate identification stage. In a few instances however, preprocessing can lead to previously viewable feature information becoming obscured. This is dependent on the skill of the person enhancing the image, which supports the opinion that a latent print examiner must first be certified. The 
exact actions taken by the latent print examiner during enhancement are detailed in the record's log file, named 'meta.docx'.

Two different processed images are included in each record. The first is the processed version of the original latent image, with no resolution changes. The second is the processed latent image, but downscaled to $1000 \mathrm{ppi}$, similarly to the other versions of the unprocessed latent image. These files are named 'study\#-processed.tiff' and 'study\#-processed-1000ppi.tiff' respectively. Examples of both image types can be seen in Figure 5.

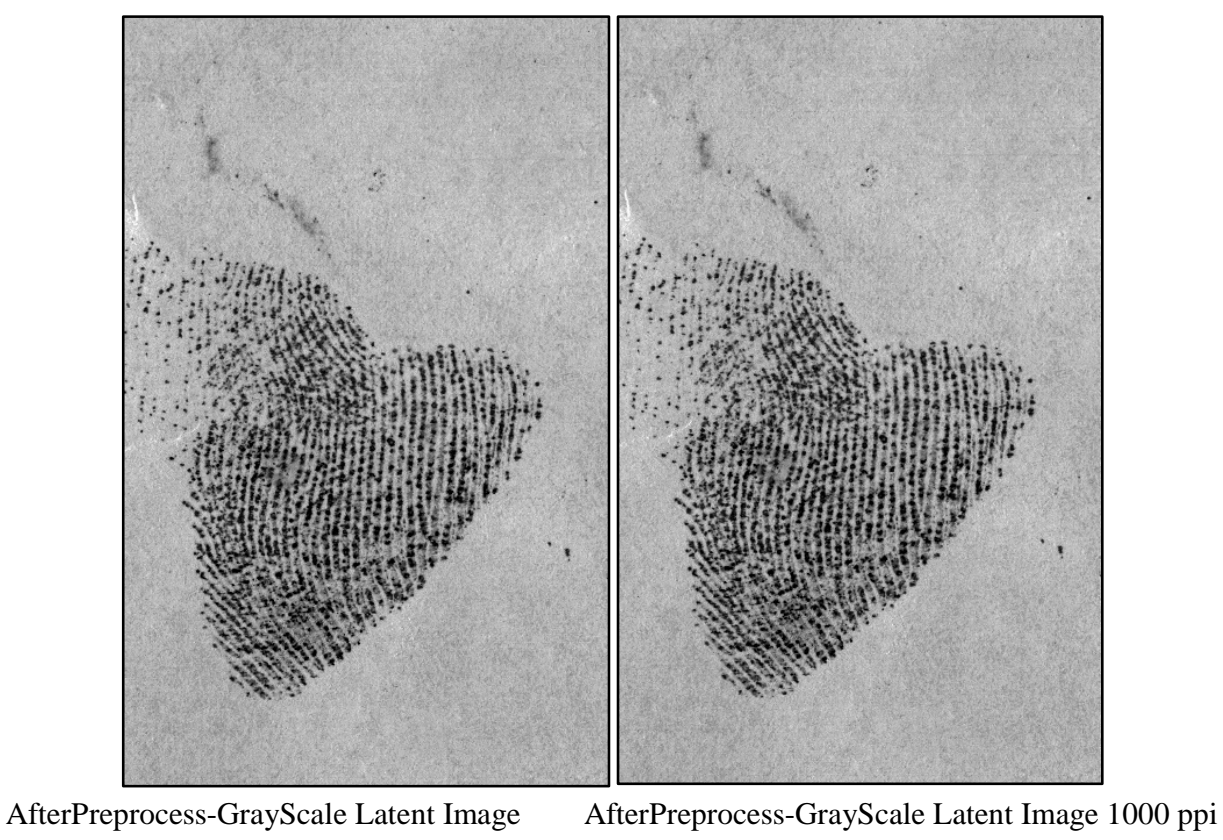

Figure 5: Record 001 Latent After Image Set

\subsubsection{FLAT/ROLLED IMAGES}

Each record is based on a single latent impression and includes the non-latent, unobscured version of that impression - the card image. They are derived from a person's fingerprint file card, which is used by FBI and other law enforcement agencies for the acquisition and retention of fingerprint impressions [17]. Card images in our database varied between 'plain' or 'rolled'. Plain fingerprint impressions, otherwise known as slap or flat impressions, are taken by pressing the subject's fingers to the live scan device. This capture method tends to generate the highest level of clarity and sequence accuracy [18]. Rolled fingerprint impressions on the other hand are collected by holding individual fingers down, and rolling them from one nail to the other, so that the entire width of the fingerprint is collected. Though rolled impressions cover the most surface area of these two capture methods, the rolling motion can introduce 
distortions or smudging if care is not taken during the collection process. The plain impressions are thus used to verify the accuracy of the rolled impressions.

The card image is saved as 'study\#-source.tiff'. The image also undergoes the same markup process as the latent files, and the resulting markup and data files are saved as 'study\#-efssource.lffs' and 'study\#-efs-source.txt'. The benchmark card file, used during the ground truth gathering process as noted in Section 2.3.4, is saved as 'study\#-benchmark-source.tiff'. Figure 6 displays examples of the card image and its feature markup file.

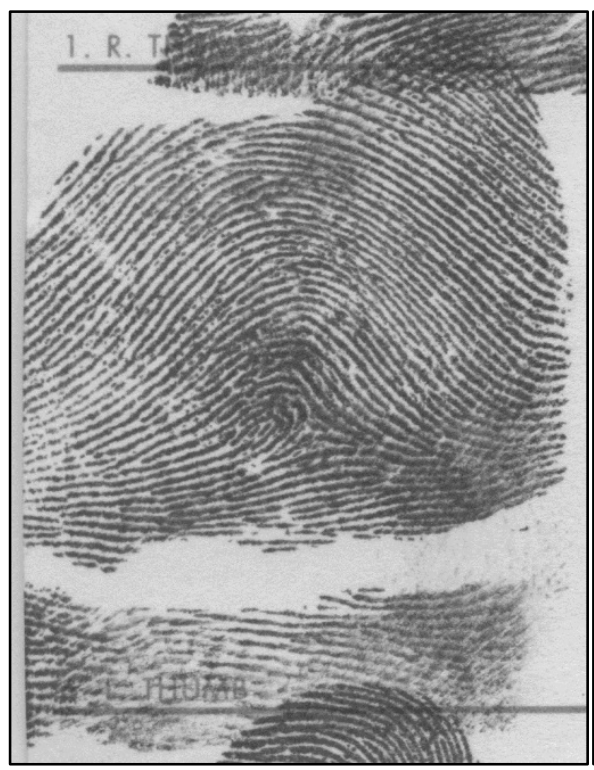

Card Image

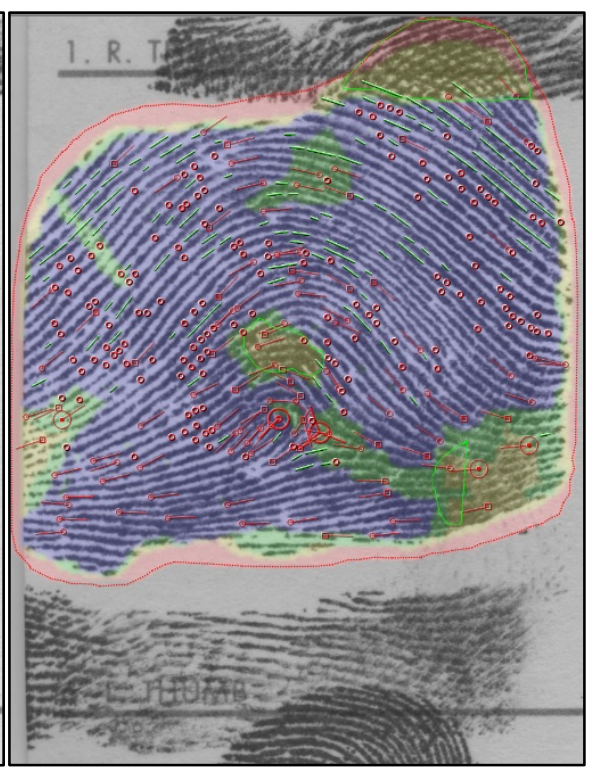

Card Image Markup

Figure 6: Record 001 Source Images

\subsubsection{BENCHMARK FILES AND ALIGNMENT}

Previously mentioned in Section 2.3.4, two benchmark images were created by examiners to estimate the rigid transformation needed to align latent to source print. To generate these benchmark images, examiners scanned the latent and card images and marked at least three shared minutiae, with each minutiae pair having the same color. Each minutia was required to be a certain distance apart from the others, to minimize rotational over-adjustment due to examiner marking error. The two benchmark images for record 001 are displayed below in Figure 7, with the colored dots emphasized. Once marked, the images were then entered into a two-part alignment calculation algorithm.

The first portion of the alignment calculation algorithm identified the locations of corresponding mark-up color points. After the images were confirmed to be in color format, 
the images were scanned to identify unique, non-grayscale colors. The pixels making up each of these colors were then isolated into a new subset that allowed for more efficient searching. From there, corresponding pixels were grouped and the center of each pixel color cluster was calculated, which represented the position of a marked minutiae.

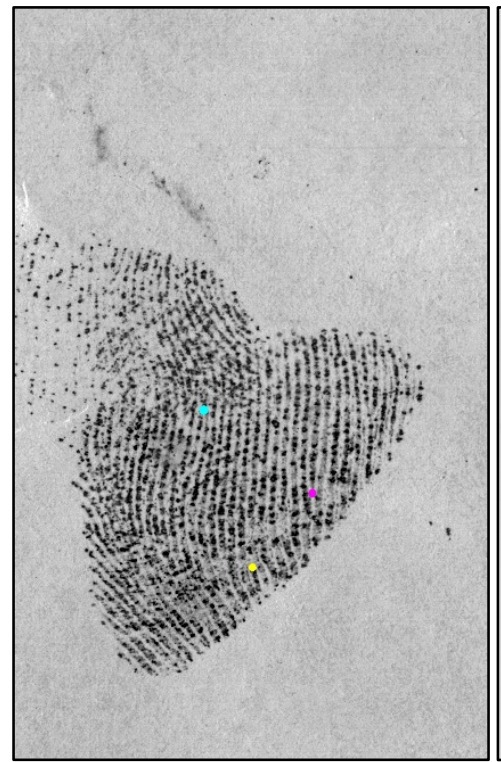

Latent Benchmark Image

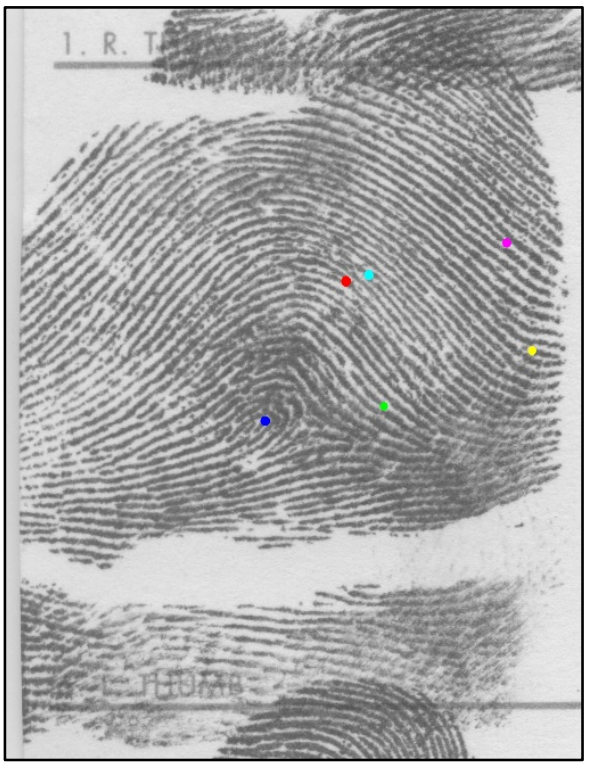

Card Benchmark Image

Figure 7: Record 001 Benchmark Images

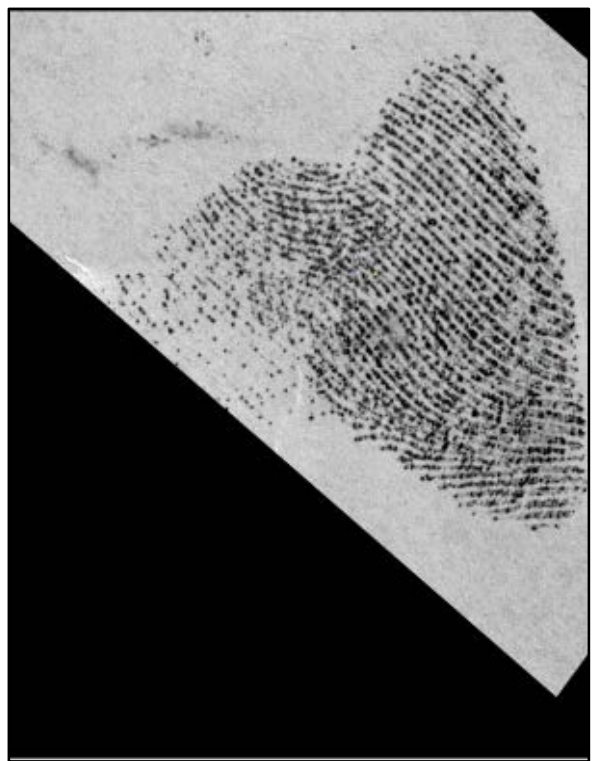

Rotated Latent

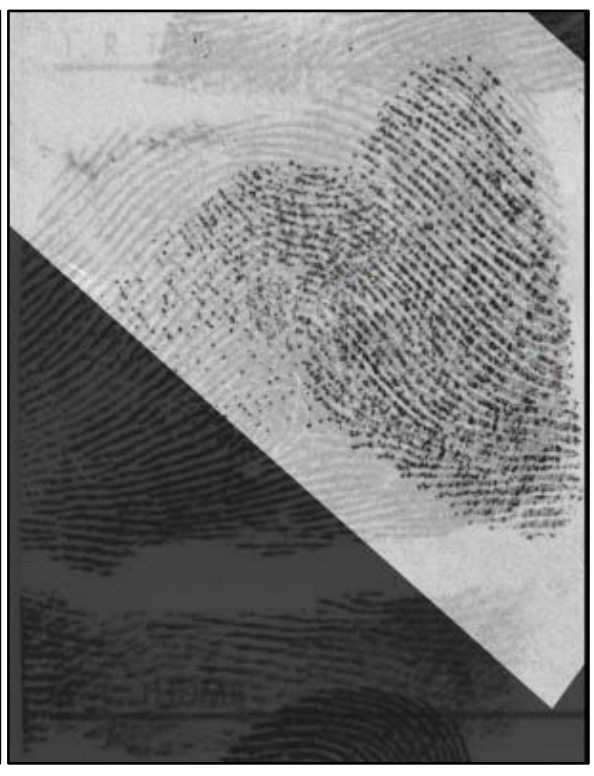

Overlaid Latent and Card

Figure 8: Record 001 Rotational Alignment Examples 
Recording the estimated centers of shared minutiae as coordinate points in the plane, the second stage of the alignment calculation algorithm implemented a least-squares algorithm to estimate the rigid transformation parameters (rotation and translation) to be applied to the latent image that results in the minimum separation distance between corresponding benchmarks. The optimum parameters are recorded as a $2 \mathrm{D}$ affine transformation matrix in the file study\#-latent-source-registration.dat. Applying this transformation to the entire latent image results in a translated and rotated latent image with the shared benchmark minutiae overlapping, though the alignment accuracy of the non-benchmark minutiae suffers due to varying levels of distortion. Examples of the isolated latent rotation and the combined benchmark image can be seen in Figure 8.

\subsubsection{MARKUP FILES}

As part of our research on the effectiveness of fingerprint preprocessing, certified latent print examiners were tasked with performing minutiae markup on a series of latent images. For each latent image, the examiners used the ULW Latent Editor software's markup tools to note features specified by EFS. These features include bifurcations, ridge endings, incipient ridges, dots, and other types of minutiae commonly used in latent friction ridge searches of Automated Fingerprint Identification System (AFIS). Examples of the marked images can be seen below in Figure 9, while the individual representations of different minutiae types can be seen in Figure 10.

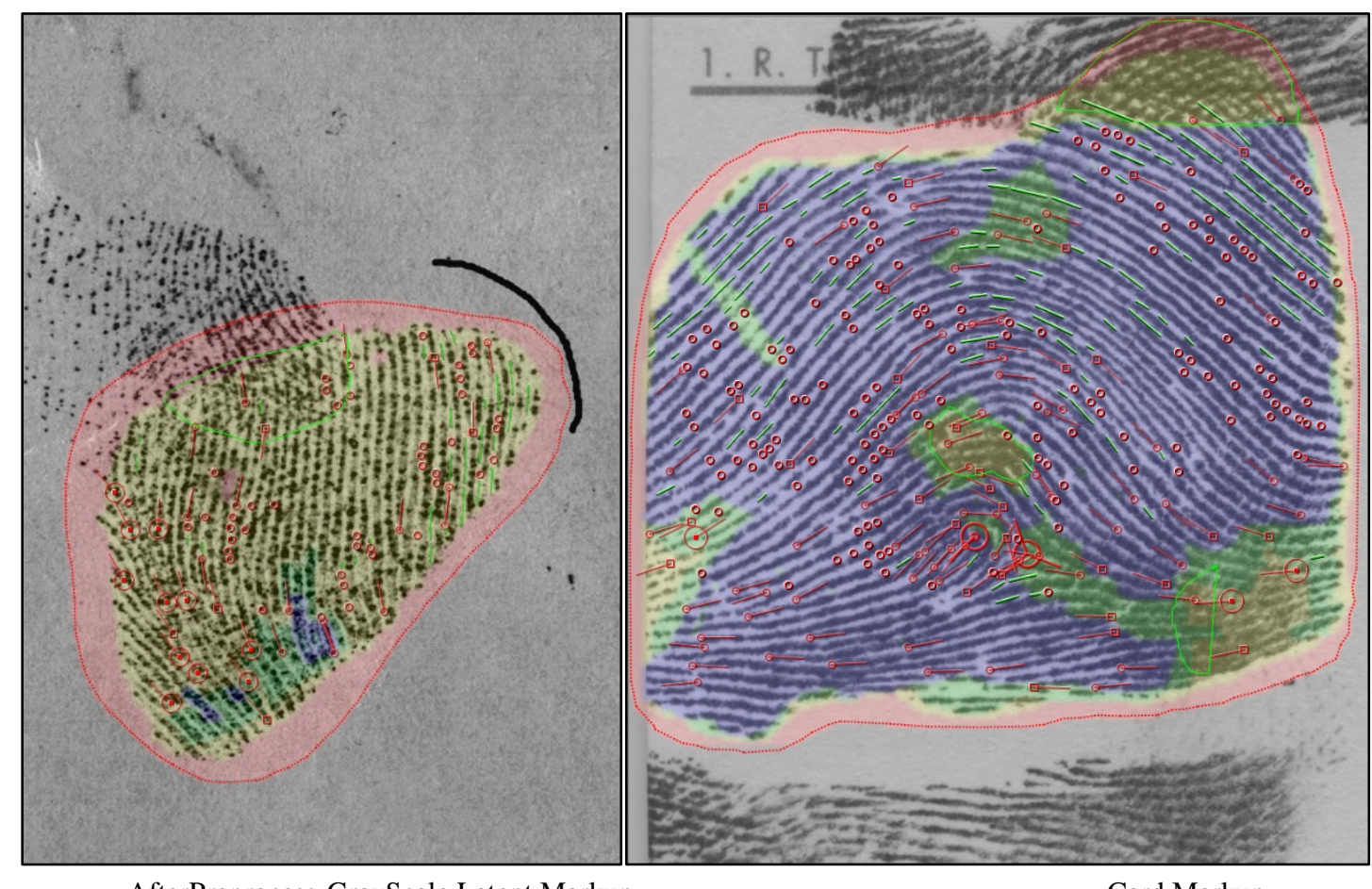

AfterPreprocess-GrayScale Latent Markup

Card Markup

Figure 9: Record 001 Latent and Card Image Markup 


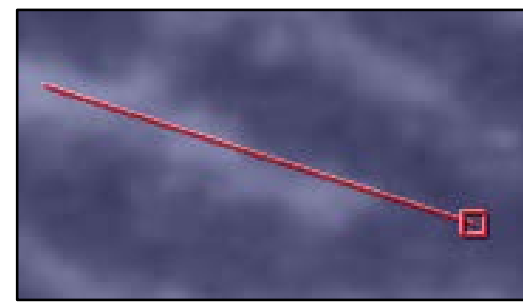

Bifurcations

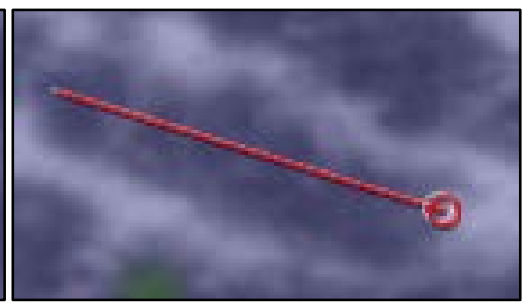

Ridge Ending

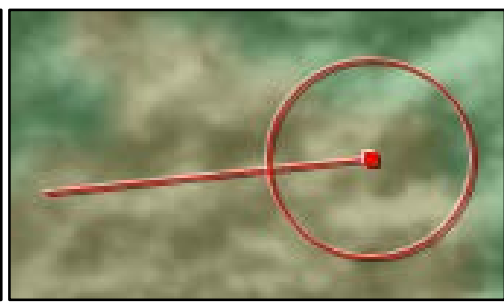

Miscellaneous Minutiae
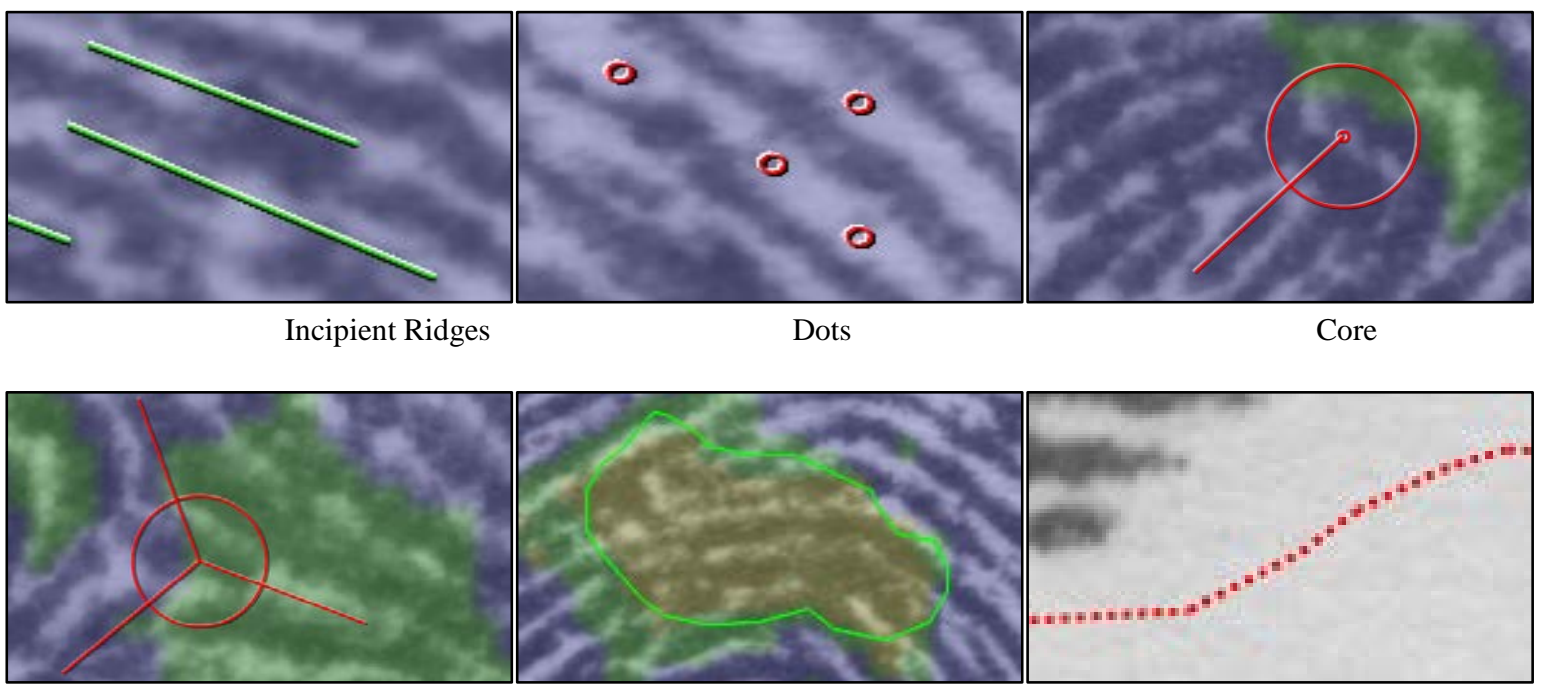

Delta

Distinctive Areas

Region of Interest

Figure 10: Minutiae Type Symbols

Bifurcations are marked with squares, ridge endings by small circles, and cores by small circles within a larger circle. Minutiae too obscure to classify are represented by lone circles of two sizes to represent the uncertainty, with higher quality unknown minutiae corresponding to the smaller of the two. The orientation for these four types of minutiae are represented by the trailing tails extending from the square, circle, or solid dot, with the angle ("theta") corresponding to the direction of the tail. Incipient ridges (also known as 'interpapillary lines' or 'Interstitial, Rudimentary or Nascent Ridges') can be seen as green lines. Dots are represented by small circles without tails, distinctive areas are outlined in a solid green line, and the region of interest is overlaid using a dotted red line. Detailed information about all marked feature information, including coordinates, thetas, uncertainty values, and more are contained in the text version of the latent search file. See section 3.1.8 for more. In total, seven EFS markup files are collected: a markup file of the card image, and two independent markup files for BeforePreprocess-GrayScale, BeforePreprocess-Color, and AfterPreprocessGrayScale latent images respectively. 
In addition to marking various minutiae, examiners also performed Latent Quality Mapping to document the subjective level of confidence in the marked features. Image quality was represented by painting over the image using the standard color definitions for latent region quality markup as defined in 'Markup Instructions for Extended Friction Ridge Features' [18] and the ANSI/NIST-ITL 1 standard [19]. As pictured in Figure 9, the color scale includes black, red, yellow, green, blue, and cyan, in order of the lowest level of confidence to the highest. Note that the markup files included in our database have black represented by unpainted areas to preserve the unmarked areas of the image. Cyan indicates that there are clear definitive ridge edges plus dots, pores and level three detail throughout the area, blue indicates that there are clear ridges, and green indicates that it is certain that every minutia in the area is marked. Note that green (or better) means that the examiner is certain of the presence of all minutiae they've marked in that region AND they are certain that there are no unmarked minutiae. Yellow indicates that the examiner is not confident in the presence or location of marked minutiae and there may be minutiae in the area that they did not mark. Finally, red indicates ridge discontinuities (e.g., smears), and black indicates the lack of ridge data in a particular area of the image.

More information about the Latent Quality Mapping color scale can be found in Section 4.1. This measurement is a focal point of the Quality Confidence Score metric (see Section 5.3), in which latent quality mapping data is quantified and compared between the BeforePreprocessGrayScale, BeforePreprocess-Color, and AfterPreprocess-GrayScale images.

\subsubsection{MARKUP METADATA FILES}

For each EFS Latent Search file, there is a corresponding text file containing detailed feature markup data, a numerical representation of the latent quality map, and extensive metadata. The metadata text files were generated using the ULW ANSINISTViewer software, which is part of the Universal Latent Workstation software package. The metadata is split into four records: transaction information, descriptive text, feature data, and latent image information. Each minutiae is recorded in the feature data section, with specific metadata fields including XY coordinates, theta, minutiae type, direction uncertainty, the radius of position uncertainty, etc., where applicable. Additionally, items of particular note in the feature include the region of interest and the quality map representation.

\section{Region of Interest}

The region of interest encompasses the area of the latent fingerprint image that holds feature information. Within the markup file, the region of interest is represented by a dotted red line that encircles the latent quality map. The width(a) and height(b) of this area are noted, as well as the vertical and horizontal offset of the region of interest. The offsets define how far from the origin the region of interest begins, with the origin being the top left corner of each latent image. The region of interest is also given as a polygon: a series of XY coordinate pairs that 
represent the exact points that the examiner used to define the region of interest. It is important to note that the region of interest size and offsets are given in micrometers, not pixels. To convert micrometers to pixels, we first convert micrometers to inches by multiplying by .000039, and then convert inches to pixels by multiplying by the resolution of the image (which is 1000 for all images used for markup).

\section{Quality Map}

To record quality map data, the latent quality map is divided into 8x8 pixel blocks. A number is assigned to each of these blocks according to what quality color is most prevalent, ranging from 0 for black/unmarked to 5 for cyan. This 1/8 scale numerical representation of the latent quality map is then recorded in the metadata file, separated by line. A comparison between the quality map in the latent markup file and the numerical representation with the metadata file can be seen below in Figure 11.

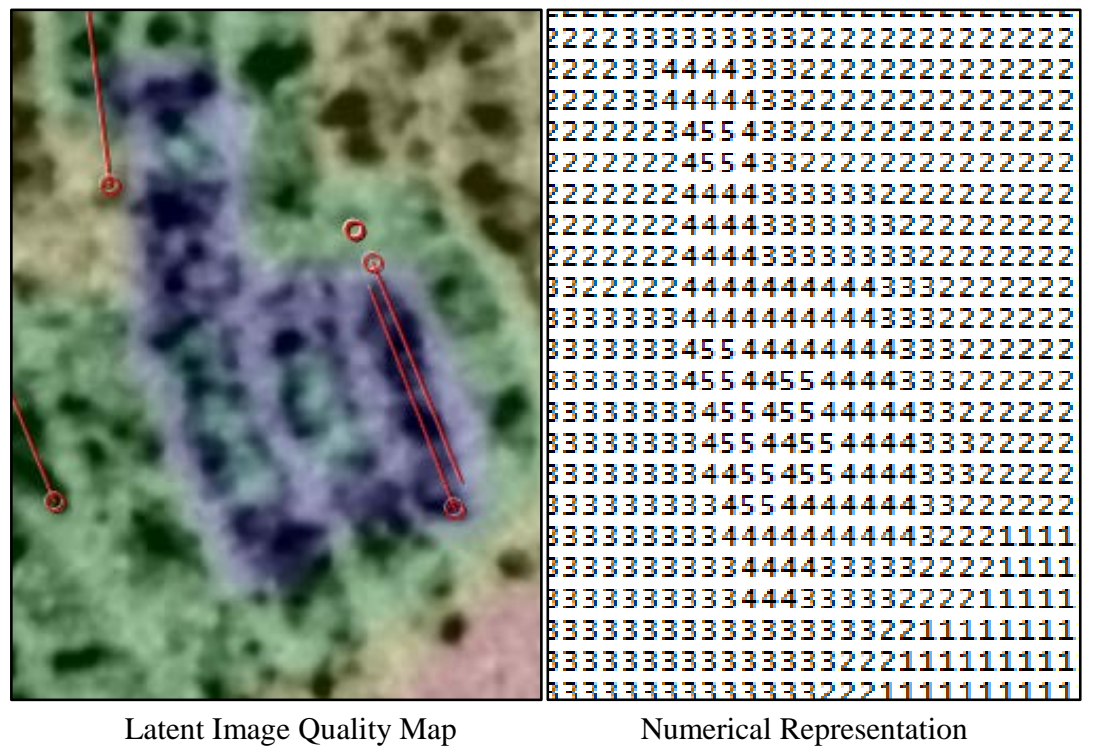

Figure 11: Quality Map Representation Comparison

\subsubsection{MASKS}

The three masks within each record are hi-contrast composite representations of the region of interests found in the EFS markup files as shown in Figure 12. The latent impression, minutiae, and other elements of the markup are removed from mask files, leaving only the region of interest indicated as a black polygon. 


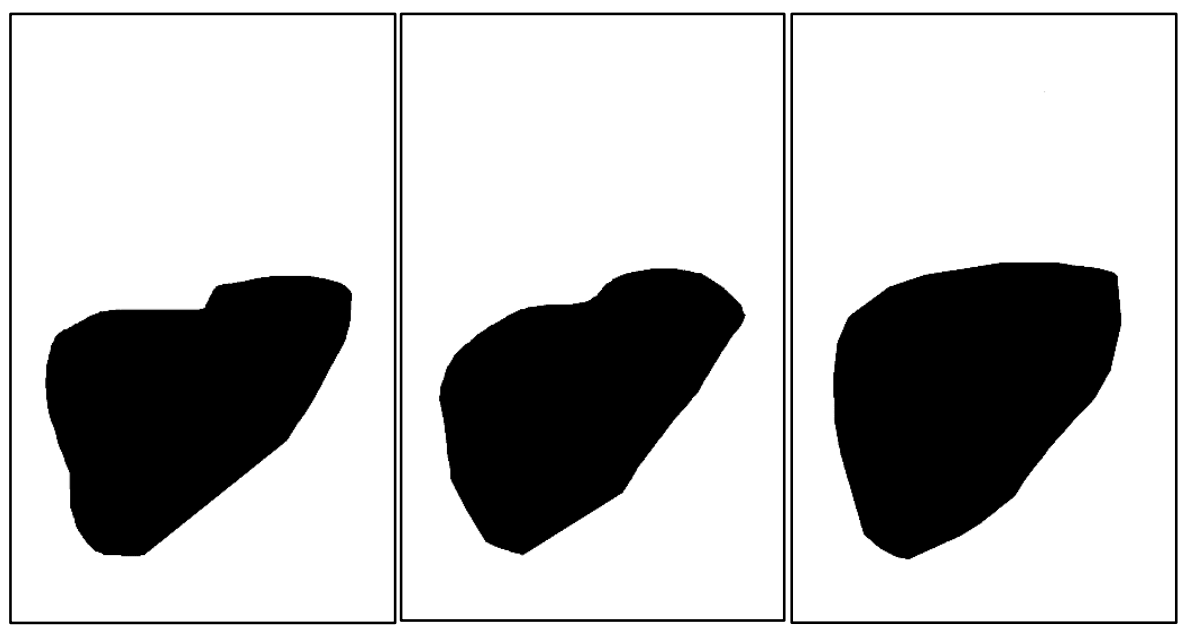

Figure 12: ROI Masks for Color, Gray, and Processed Latent Images

\subsubsection{MATCHED LATENT AND PROCESSED}

In this study we quantify latent information in terms of minutiae. Thus, an elementary matching algorithm is required which takes as its input a pair of EFS marked images and returns a list of corresponding minutiae on output. Some amount of location variability is to be expected in identifying this correspondence. However, no rotational nor other distortions are considered as the images within a record are distinguished by image processing treatments, for example Color or Grayscale, but are otherwise identical. In more detail, the algorithm first pulls the metadata of the minutiae contained within both markup files, along with resolution and Dots Per Inch (DPI) information. It then loops over the minutiae list in the first image searching for any minutiae in the second image that lie within a circular region with a radius of 20 pixels. Any minutiae in the second image that are found within this basin are compared considering distance from the first, and minutiae type. Based on this comparison, minutiae between the image pairs are either identified as matched, or as standalone. Matched minutiae have their coordinate positions averaged to create a consensus estimate of its location.

An example of a matchup image across a combined BeforePreprocess-Color point set and the corresponding combined AfterPreprocess-GrayScale image point set can be seen below in Figure 13. The two comparison sets of minutiae are marked as red and blue letters, with the specific type of minutiae indicated by the letter. The 20-pixel match requirement radius is represented by a while circle. If a match is successfully found, the two minutiae are linked by a green line. Three visual comparison images are included in the database - one that compares BeforePreprocess-GrayScale to AfterPreprocess-GrayScale, one that compares BeforePreprocess-Color to AfterPreprocess-GrayScale, and one that compares BeforePreprocess-GrayScale to BeforePreprocess-Color. 


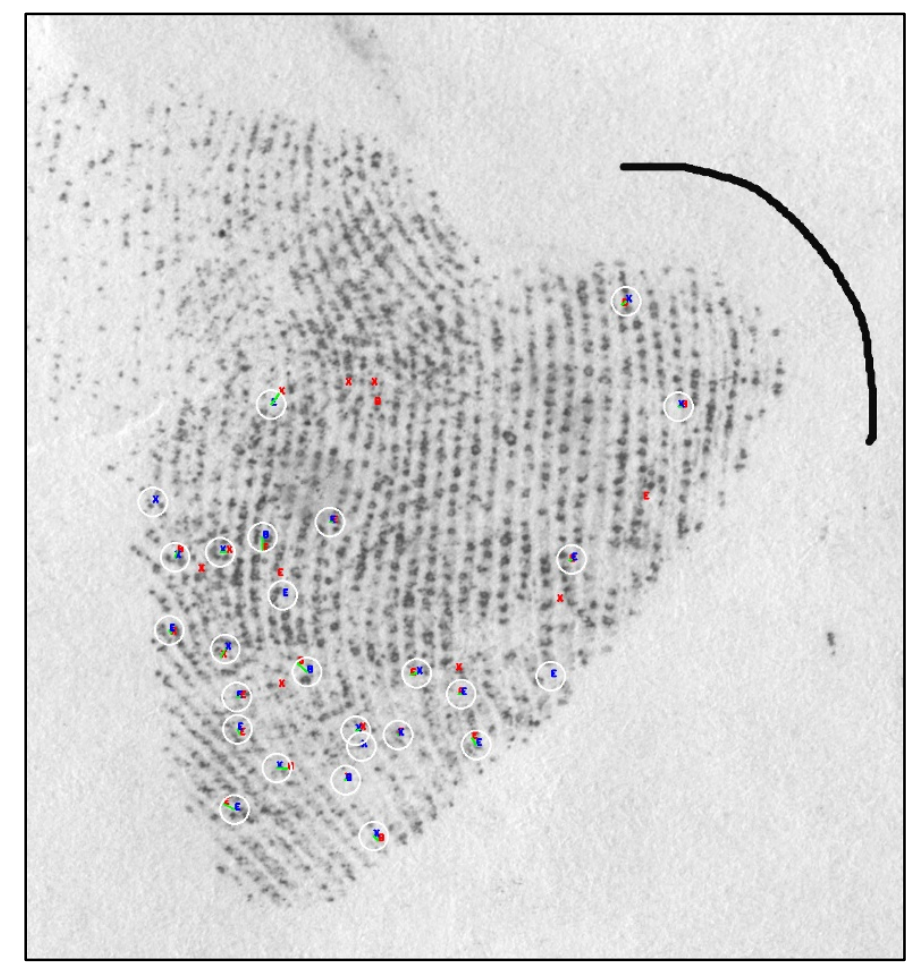

Figure 13: Matched Minutiae for Color Latent and Processed Images

\subsubsection{METADATA FILES}

In addition to the latent images and markup files, there are six files in each record dedicated to recording metadata, ranging from information about the acquisition process to the dimensions of the fingerprint polygon. The contents of the multi-item metadata file (-info.csv) can be seen below with definitions for each category within. Single item metadata files are grouped in the Misc. Metadata File Contents table.

Table 2: Info.csv File Categories and Definitions

\begin{tabular}{|l|l|}
\hline -info.csv & Description \\
\hline Category Name & The finger within the latent image. \\
& 1 - Right Thumb 2 - Right Index \\
& 3 - Right Middle 4 - Right Ring \\
Source Finger \# & 5 - Right Pinky 6 - Left Thumb \\
& 7 - Left Index 8 - Left Middle \\
& 9 - Left Ring 10 - Left Pinky \\
& 11 - Unusable \\
\hline
\end{tabular}




\begin{tabular}{|c|c|}
\hline Latent Acquisition Procedure & $\begin{array}{l}\text { The specific lifting procedure used to procure the latent } \\
\text { fingerprint image. Options include Bi-Chromatic Mag } \\
\text { Powder Developed Prints, Bi-Chromatic Powder Developed } \\
\text { Prints, Black Ink Pad on Colored Background, Ninhydrin } \\
\text { Developed Prints, Silver Mag Powder Developed Prints, and } \\
\text { White Powder Developed Prints. }\end{array}$ \\
\hline Good, Bad, Ugly (GBU) of Latent & $\begin{array}{l}\text { The quality rating assigned to the latent (BeforePreprocess- } \\
\text { Color) image by an examiner, using GBU scale. }\end{array}$ \\
\hline GBU of Processed & $\begin{array}{l}\text { The quality rating assigned to the processed } \\
\text { (AfterPreprocess-GrayScale) image by an examiner, using } \\
\text { GBU scale. }\end{array}$ \\
\hline Enhancement ID & $\begin{array}{l}\text { Signifies how many times the latent image was re-lifted } \\
\text { using different scanning hardware. }\end{array}$ \\
\hline Examiner ID: Latent Gray - 1 & $\begin{array}{l}\text { The ID number (1-9) of the latent examiner responsible for } \\
\text { the EFS markup of 'study\#\#\#-efs-latent-gray-1.lffs'. }\end{array}$ \\
\hline Examiner ID: Latent Gray - 2 & $\begin{array}{l}\text { The ID number (1-9) of the latent examiner responsible for } \\
\text { the EFS markup of 'study\#\#\#-efs-latent-gray-2.lffs'. }\end{array}$ \\
\hline Examiner ID: Latent Color - 1 & $\begin{array}{l}\text { The ID number (1-9) of the latent examiner responsible for } \\
\text { the EFS markup of 'study\#\#\#-efs-latent-color-1.lffs'. }\end{array}$ \\
\hline Examiner ID: Latent Color - 2 & $\begin{array}{l}\text { The ID number (1-9) of the latent examiner responsible for } \\
\text { the EFS markup of 'study\#\#\#-efs-latent-color-2.lffs'. }\end{array}$ \\
\hline Examiner ID: processed - 1 & $\begin{array}{l}\text { The ID number (1-9) of the latent examiner responsible for } \\
\text { the EFS markup of 'study\#\#\#-efs-processed-1.lffs'. }\end{array}$ \\
\hline Examiner ID: processed - 2 & $\begin{array}{l}\text { The ID number (1-9) of the latent examiner responsible for } \\
\text { the EFS markup of 'study\#\#\#-efs-processed-2.lffs'. }\end{array}$ \\
\hline Gray/Color 1 Resolution & $\begin{array}{l}\text { The resolution (either } 1000 \text { ppi or } 1200 \mathrm{ppi} \text { ) of 'study\#\#\#- } \\
\text { efs-latent-gray-1.lffs' and 'study\#\#\#-efs-latent-color-1.lffs' }\end{array}$ \\
\hline Gray/Color 2 Resolution & $\begin{array}{l}\text { The resolution (either } 1000 \text { ppi or } 1200 \text { ppi) of 'study\#\#\#- } \\
\text { efs-latent-gray-2.lffs' and 'study\#\#\#-efs-latent-color-2.lffs' }\end{array}$ \\
\hline Processed 1 Resolution & $\begin{array}{l}\text { The resolution (either } 1000 \text { ppi or } 1200 \text { ppi) of 'study\#\#\#- } \\
\text { efs-processed-1.lffs'. }\end{array}$ \\
\hline Processed 2 Resolution & $\begin{array}{l}\text { The resolution (either } 1000 \text { ppi or } 1200 \text { ppi) of 'study\#\#\#- } \\
\text { efs-latent-color-2.lffs'. }\end{array}$ \\
\hline
\end{tabular}


Table 3: Misc. Metadata File Categories and Definitions

\begin{tabular}{|c|c|}
\hline \multicolumn{2}{|l|}{ Misc. Metadata Files } \\
\hline Category & Description \\
\hline Latent-source-registration.dat & $\begin{array}{l}\text { The } 3 \times 3 \text { transformation matrix used to align the latent } \\
\text { image with the card image. }\end{array}$ \\
\hline Meta.docx & $\begin{array}{l}\text { A record of examiner actions performed during } \\
\text { preprocessing. }\end{array}$ \\
\hline Poly.txt & $\begin{array}{l}\text { Contains the coordinate pairs that make up polygonal } \\
\text { representation of the latent impression. }\end{array}$ \\
\hline & $\begin{array}{l}\text { Contains the coordinate pairs that make up the } \\
\text { rectangular region of interest. }\end{array}$ \\
\hline Roi.txt & $\begin{array}{l}\text { Ex. An ROI of }[2,280,545,876] \text { would be the region } \\
\text { with an upper left-hand corner of } 2,280 \text { and a lower } \\
\text { right-hand corner of } 545,876 \text {. }\end{array}$ \\
\hline
\end{tabular}

\section{MEASUREMENTS}

\subsection{QUALITY MAP SCALE}

The EFS quality maps are generated by LPEs following a strict assessment guideline as defined in the NIST Special Publication - Markup Instructions for Extended Ridge Features [18]. Different color grades are used to document the perceived clarity of features in a particular area of the latent image. The grades can also signify a level of confidence in absent features. For example, if an area of the latent image is marked as high quality but there are no marked features, that indicates that the examiner is confident that there is no feature information present in that location.

When marking quality areas, examiners follow a strict flowchart of questions, starting with 'Is any ridge information present?' If no, the area is marked black - which can be represented as areas with no color as shown in Figure 14. Areas outside of the region of interest, additional 
fingerprints, and other surrounding details are all considered to have no valuable ridge impression information.
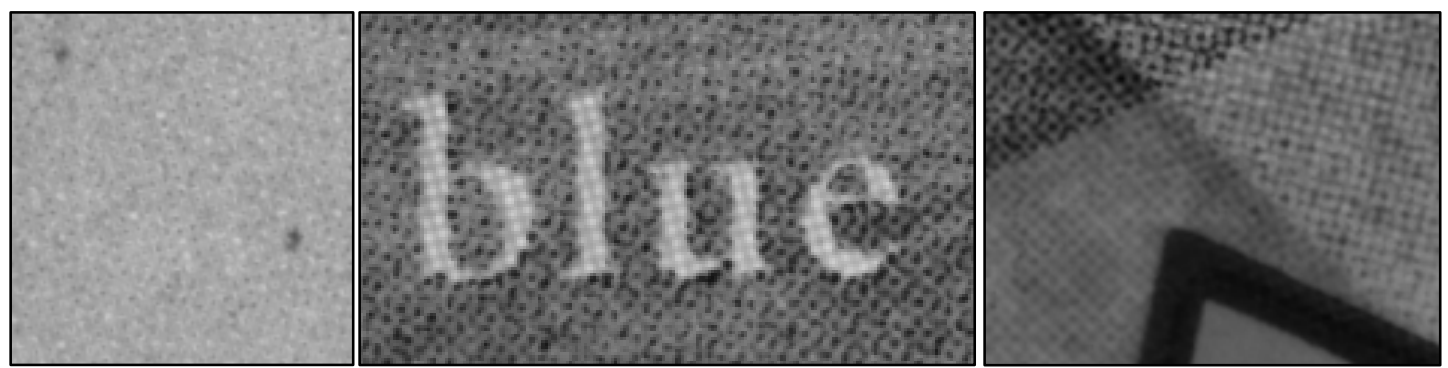

Figure 14: Examples of "Black" areas in latent quality region mark-up

If ridge information is present, examiners then move onto 'Are you certain of the continuity of ridge flow?' If no, the area as shown in Figure 15 is marked red. These areas may have ridge data, but the quality of the area makes that data unsuitable for reliable comparison.

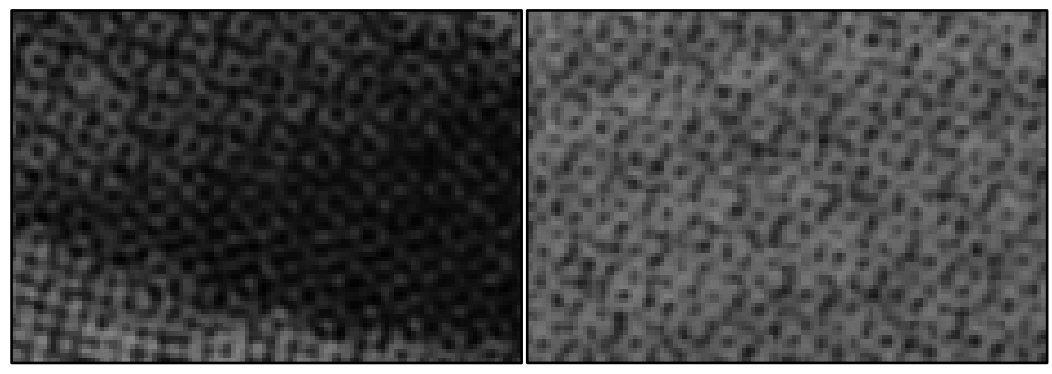

Figure 15: Examples of "Red" areas in latent quality region mark-up

The next question in the quality determination flowchart is 'Are you certain of the location, presence, and absence of all minutiae?' Areas that do not pass this stage as shown in Figure 16 are marked yellow, indicating that ridge flow data is certain, but minutiae types and positions cannot be guaranteed.

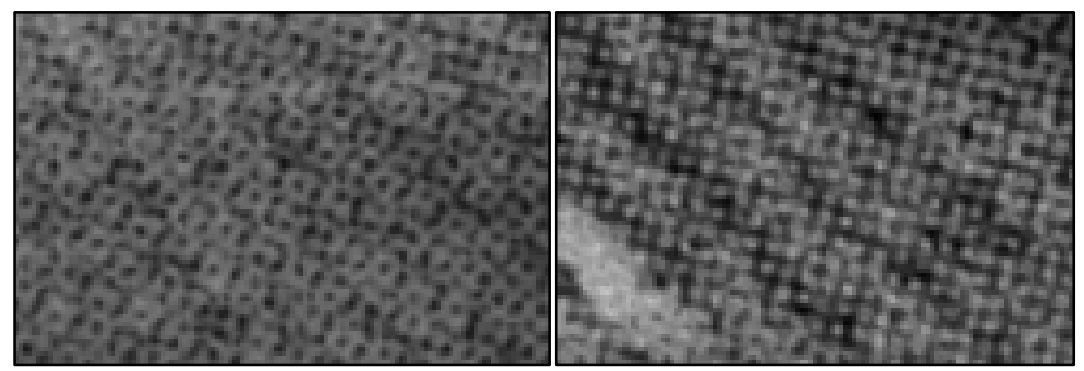

Figure 16: Examples of "Yellow” areas in latent quality region mark-up 
If minutiae locations are guaranteed, then examiners consider 'Are the ridge edge contours clear and unambiguous?' If not, the areas as shown in Figure 17 are marked green. Minutiae marked in green and higher quality areas are almost completely accurate in both position and existence.

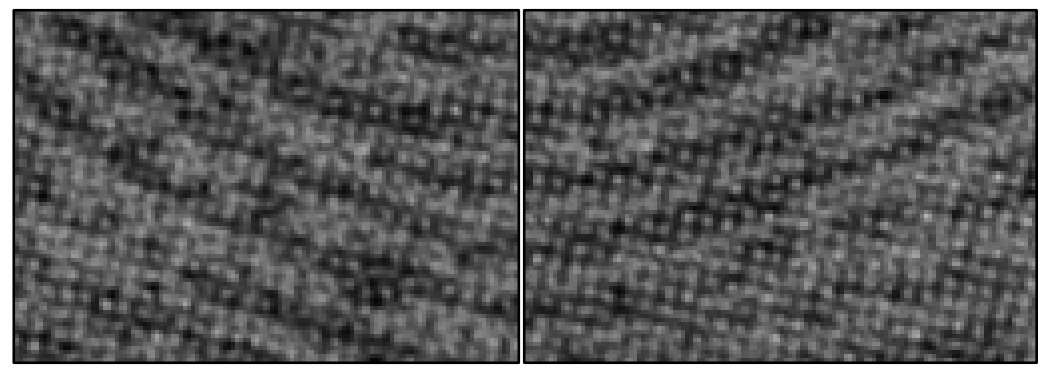

Figure 17: Examples of “Green” areas in latent quality region mark-up

After examining the ridge edge contours, examiners next look at if the pores are 'clear and unambiguous'. If the quality check fails this final step, the area as shown in Figure 18 is marked blue. Blue areas are considered to have 'definitive ridge quality', with 'all levels of ridge features other than the pores not only visible or interpretable but obvious and unambiguous, including the shape and contours of ridge edges, and the shape and precise location of all minutiae, dots, and incipients.'
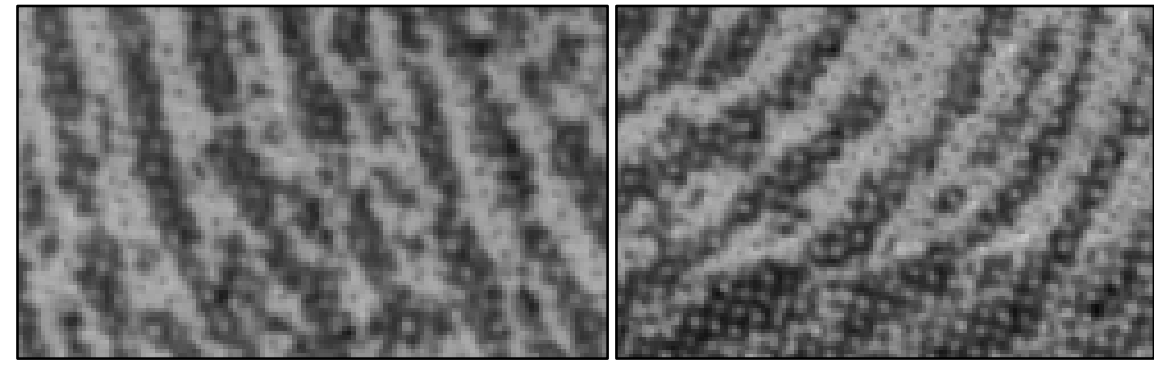

Figure 18: Examples of “Blue” areas in latent quality region mark-up

However, if the quality of the area holds up to the final pore evaluation, the area as shown in Figure 19 is marked as teal. Teal level quality areas are considered to be the highest quality and are exceedingly rare in unprocessed latent images. 


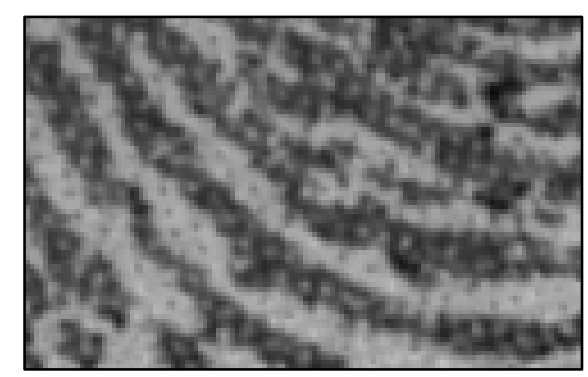

Figure 19: Examples of “Teal” areas in latent quality region mark-up

Additional information about the ridge quality map and feature markup can be found in the ANSI/NIST standard [19].

\subsection{GBU LATENT VALUES}

Alongside marking minutiae locations and quality areas, the LPEs were instructed to grade the overall quality value of the image using the 'Good, Bad, and Ugly' (GBU) scale [15]. Figure 20 - Figure 22 show examples of each quality value assessment, with both the original image and the corresponding quality map.
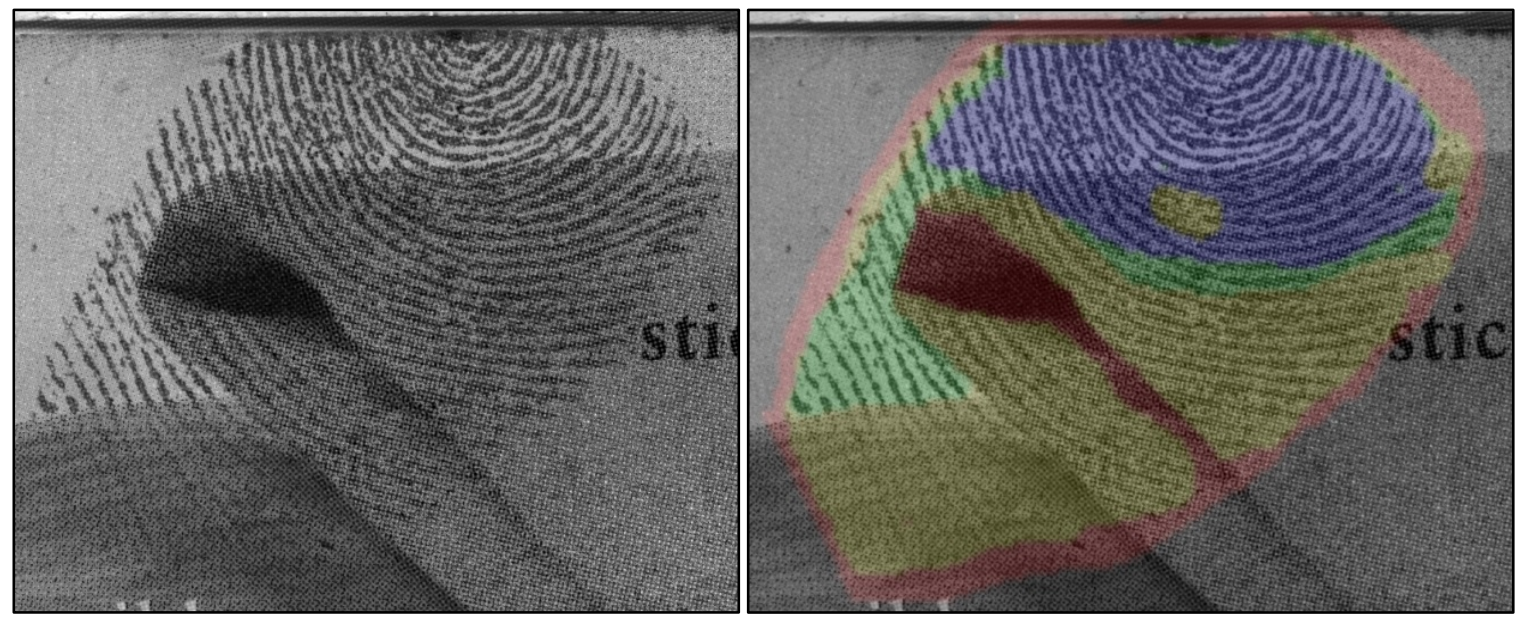

Figure 20: Good Quality Latent Images 


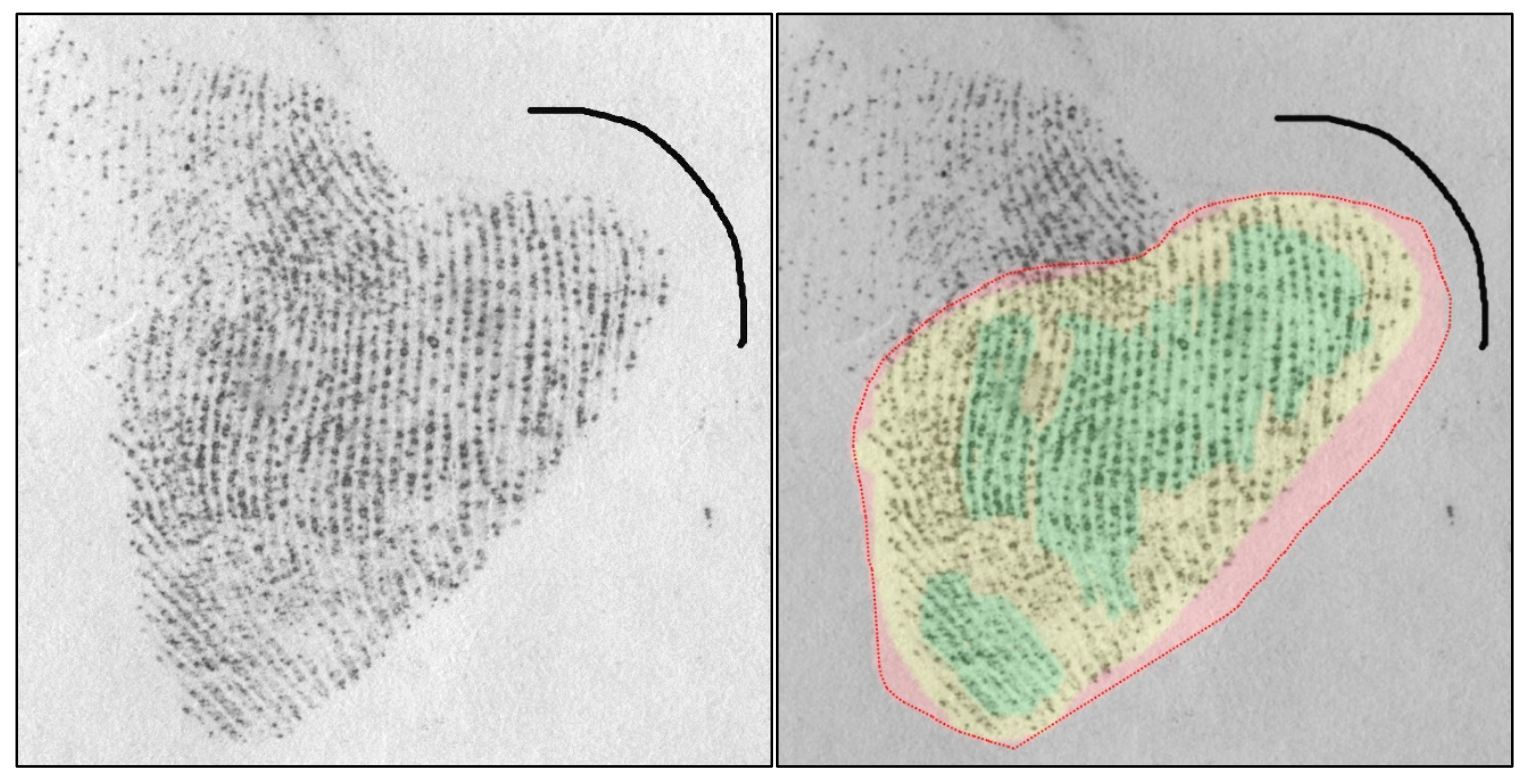

Figure 21: Bad Quality Latent Images

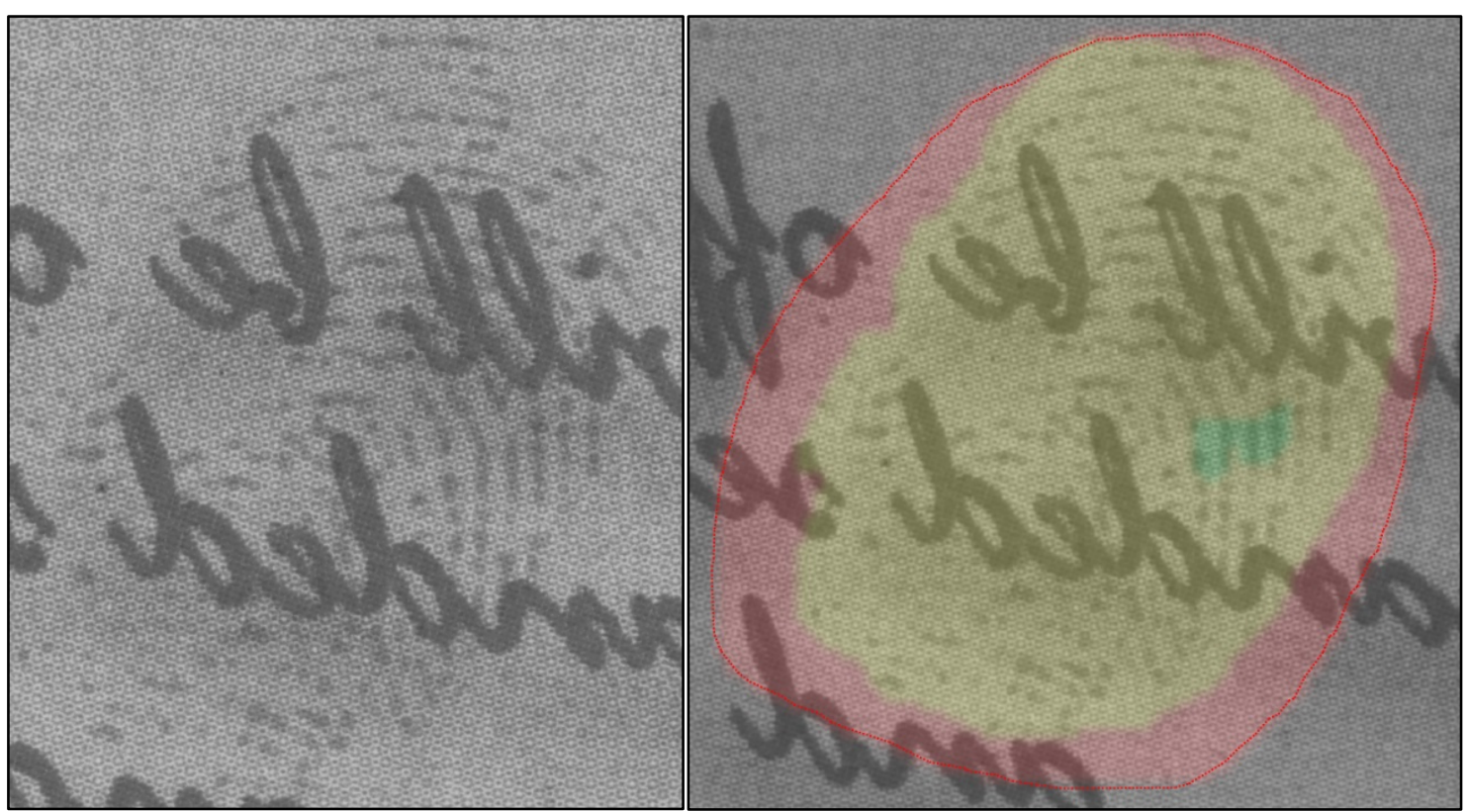

Figure 22: Ugly Quality Latent Images 


\subsection{INTERSECTION SET CALCULATION}

With two examiners performing the EFS markup for the BeforePreprocess-GrayScale, BeforePreprocess-Color, and AfterPreprocess-GrayScale, the final dataset ends up having two copies of potential minutiae coordinates. To calculate minutiae-based quality metric improvements, it was necessary to create an algorithm to consolidate the two minutiae sets for each phase into a single intersecting set. In the previous publication of this data [6], we presented the union set of minutiae, minus any duplications. In this report, we present the intersection set of minutiae.

After extracting the minutiae coordinate data from the EFS text files, the resolution data from each record's Info.csv file was pulled. The DPI data was used in conjunction with the saved coordinate position to covert the micrometer value of the coordinate to a standard pixel value. These new, pixel-based coordinates were then sent over to a correspondence script, in which minutiae from one examiner set were compared to minutiae from the other examiner's set. Search areas for each minutia were set to 20 pixels in each direction, and any minutiae from the other examiner set were sorted into a potential match set. The potential matches were then vetted for minutiae type, with match order going in favor of those minutiae that were in the search area and shared the same type. This matching process looped through all 6 sets of minutiae location points to create the dataset that we base our minutiae gain percentage metric analysis on, as seen in Section 5.1.

\section{QUALITY METRICS}

The quality metrics represented here were based on the 89 sample records in the accompanying database. The significance and efficacy of preprocessing was determined by the analysis of changes in three quality metrics: minutiae gain percentage, image value determination, and the composite quality confidence score. 


\subsection{MINUTIAE GAIN PERCENTAGE}

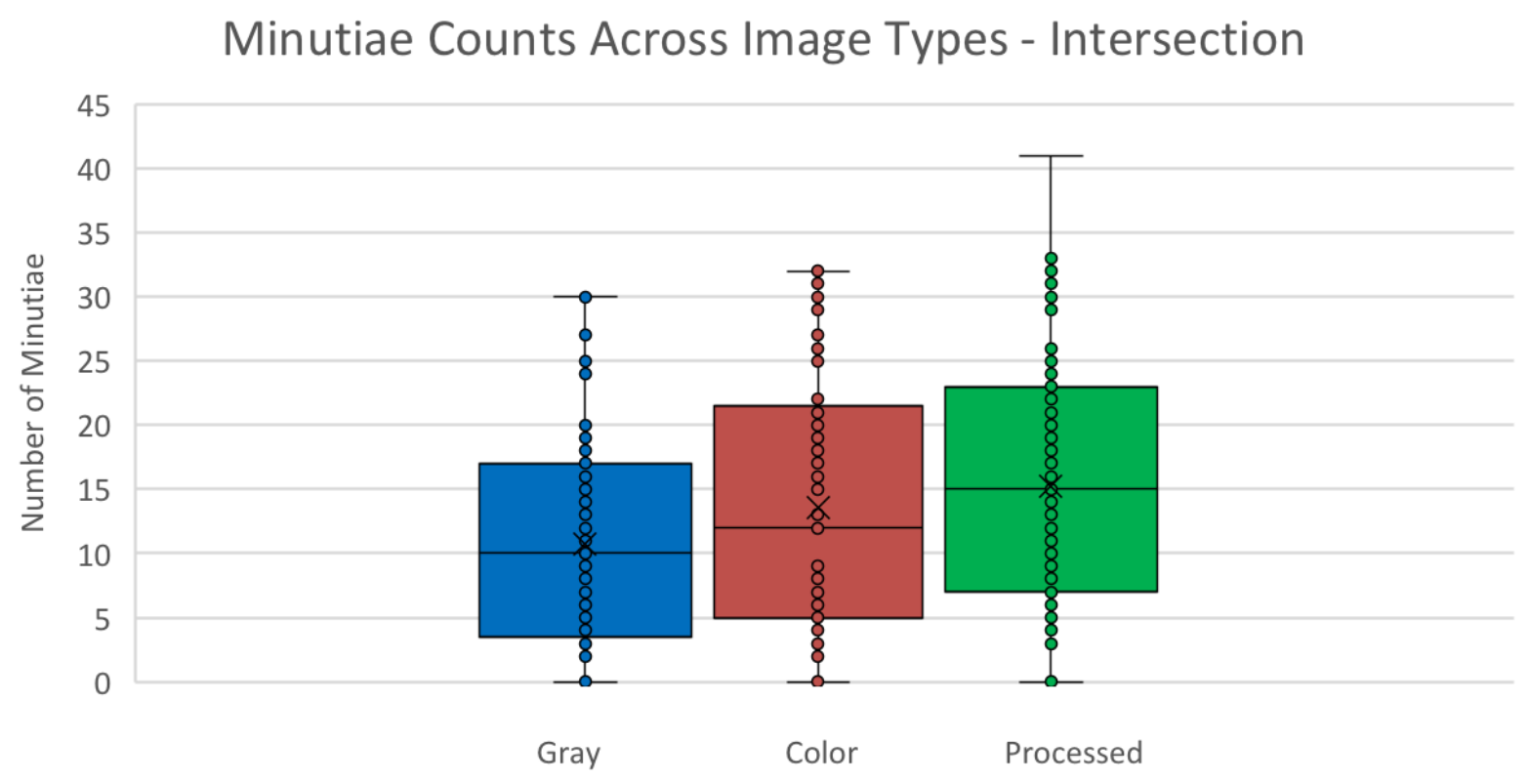

Figure 23: Minutiae Count

The minutiae gain percentage is based on the intersection set of the 6 EFS markups (2 for each: BeforePreprocess-GrayScale, BeforePreprocess-Color, and AfterPreprocess-GrayScale). The combined dataset calculation method is detailed in Section 4.3.

Overall minutiae feature count across image types is shown in Figure 23. There was an average of 10.73 minutiae in the BeforePreprocess-GrayScale images, 13.58 minutiae in the BeforePreprocess-Color images, and 15.25 minutiae in the AfterPreprocess-GrayScale images.

Given the wide range of minutiae counts across the 89 data records, we chose to analyze within-record changes normalized as gain percentages as shown in Table 5 . The minutiae gain percentage for images of two types is defined as the increase in minutiae count divided by the initial (lower) count. For example, to compare a BeforePreprocess-GrayScale image to the AfterPreprocess-GrayScale image (i.e., the preprocessed image), the percentage gain is (\# After - \# Before)/(\# Before), where the number sign indicates \# of minutiae. We then computed the mean and median gain percentages across the entire dataset from this series of percentages $(\mathrm{N}=89)$. 
Table 5: Minutiae Gain Percentage (where BG represents BeforePreprocess-GrayScale, BC represents BeforePreprocess-Color, and AG represents AfterPreprocess-GrayScale)

\begin{tabular}{|l|l|l|}
\hline Image Comparison & Median & Mean \\
\hline BG to BC & $18.18 \%$ & $29.16 \%$ \\
\hline BG to AG & $33.33 \%$ & $77.26 \%$ \\
\hline BC to AG & $19.26 \%$ & $46.86 \%$ \\
\hline
\end{tabular}

The largest median gain comes from comparing BeforePreprocess-GrayScale images to their processed counterparts. The BeforePreprocess-Color images appear to sit roughly half-way between this information gain, in the sense that percentages BG-to-BC and BC-to-AG are roughly equal and approximately sum to BC-to-AG. The large differences between median and mean increases for the images can be attributed to a handful of cases where there were large $(>=200 \%)$ differences in detected minutiae. There were 13 such cases in the BeforePreprocess-GrayScale to AfterPreprocess-GrayScale comparison, 10 such cases in the BeforePreprocess-Color to AfterPreprocess-GrayScale comparison, and 6 such cases in the BeforePreprocess-GrayScale to BeforePreprocess-Color comparison. The gain percentage distribution for the three comparisons can be seen below in Figure 24. 


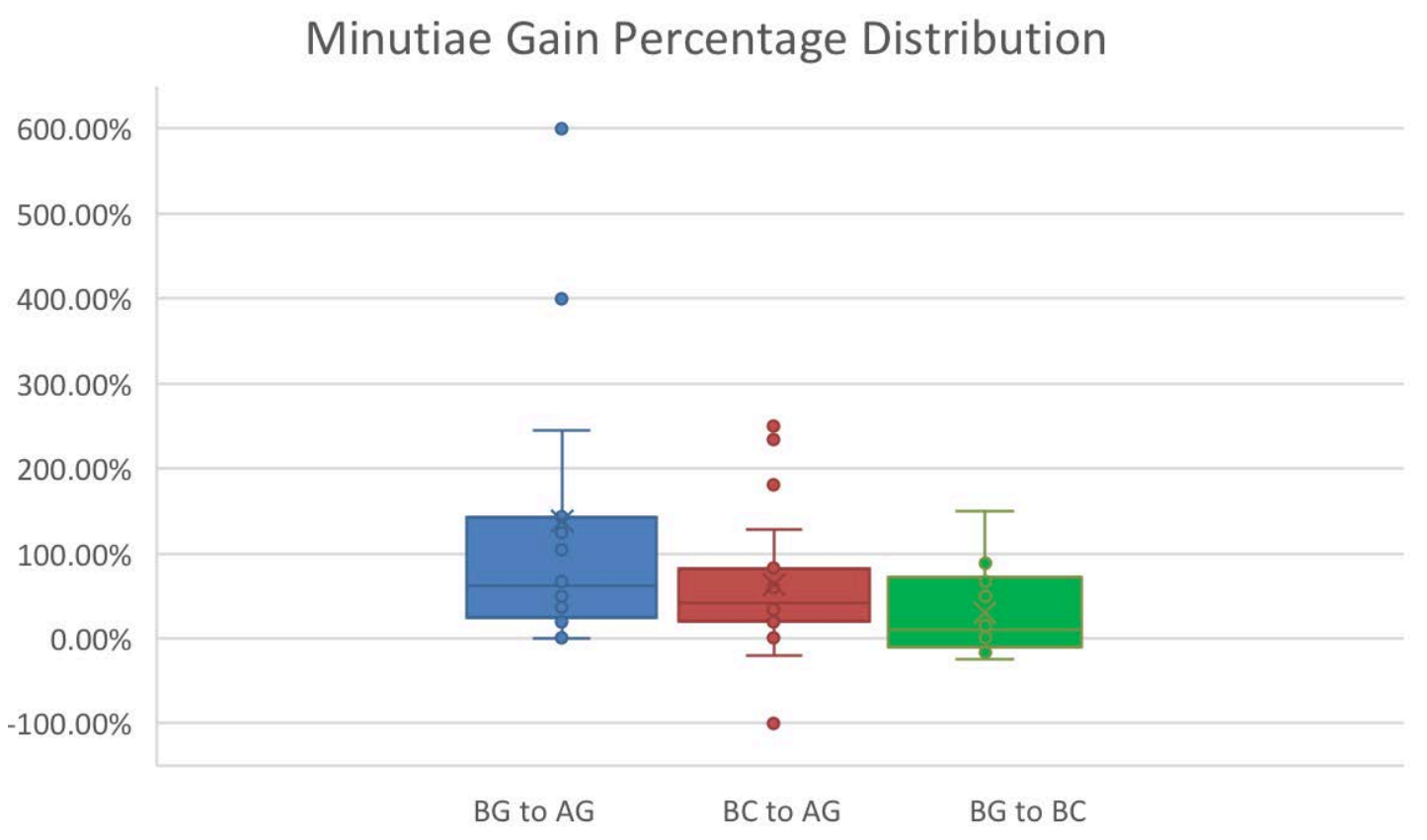

Figure 24: Minutiae Gain Percentage Distribution

The Wilcoxon Signed Rank Test [20] was used to test the statistical significance between the minutiae count differences. Given a paired list of minutiae counts, this nonparametric test computes a score by: 1 . rank ordering the absolute value of all differences, 2 . reassigning the sign of the difference to the ranked list, and 3. evaluating the signed rank sum $(W)$. Under the null hypothesis that the minutiae count distribution is the same between the two groups, $W$ will be close to zero. As all sample sizes are greater than $10, W$ may be approximated by a normal random variable. We calculate the z-value by dividing the test statistic $(W)$ by the standard deviation of its sampling distribution $(\sigma w)$. This standard deviation is computed as the square root of $\left(N_{r}\left(N_{r}+1\right)\left(2 N_{r}+1\right)\right) / 6$, where $N_{r}$ is the sample size.

$$
z=\frac{W}{\sigma w}, \sigma w=\sqrt{\frac{N_{r}\left(N_{r}+1\right)\left(2 N_{r}+1\right)}{6}}
$$

Based on the $\mathrm{z}$-value, we can determine the two-tailed probability score $\mathrm{P}(<0.05)$. For a more extended explanation regarding the method and the individual steps, see [20].

As part of the significance testing for the minutiae count differences, three comparisons were performed. Note that the different values for $N_{r}$ are the result of image pairs with no change in the number of minutiae features identified. In these cases, the record was not included in the significance analysis. The results can be seen below in Table 6 . 
Table 6: Wilcoxon Signed Ranks Test Results - Minutiae Count (where BG represents BeforePreprocess-GrayScale, BC represents BeforePreprocess-Color, and AG represents AfterPreprocess-GrayScale)

\begin{tabular}{|l|l|l|l|l|}
\hline Comparison & $\mathbf{N}$ & $\begin{array}{l}\text { Test } \\
\text { Statistic(W) }\end{array}$ & $\mathbf{Z}$ & $\mathbf{P}$ \\
\hline BG to BC & 77 & -2690 & -6.8292 & $<.0001$ \\
\hline BG to AG & 80 & -2691 & -6.4534 & $<.0001$ \\
\hline BC to AG & 82 & -1325 & -3.0627 & .0022 \\
\hline
\end{tabular}

The first comparison, BeforePreprocess-GrayScale to BeforePreprocess-Color, was done to measure any marked improvement in feature information between the starting samples. Using an $\mathrm{N}$ of 77, the comparison results in a test statistic of -2690 and a z-score of -6.8292, which lead to a p-value of <.0001. The second comparison, BeforePreprocess-GrayScale to AfterPreprocess-GrayScale, was performed to measure improvement in feature information due to preprocessing. Using an $\mathrm{N}$ of 80, the comparison results in a test statistic of -2691 and a z-score of -6.4534 , which lead to a p-value of <.0001. The third and final comparison, BeforePreprocess-Color to AfterPreprocess-GrayScale, measures the ability to BeforePreprocess-Color image to preserve feature information. Using an $\mathrm{N}$ of 82, the comparison results in a test statistic of -1325 and a z-score of -3.0627, which lead to a p-value of .0022. With $\alpha=.05$, the Wilcoxon Signed Ranks Test indicate that the results of the three comparisons are statistically significant. We can then conclusively say that more feature information is preserved in the BeforePreprocess-Color latent image than its grayscale counterpart, and that preprocessed images contain more feature information than either of the Before images. In addition to the Wilcoxon Signed Ranks Test, we also tested for significance using a Random Matched Sample analysis, which confirmed the results documented here.

Currently, the most commonly used AFIS systems and matching tools are limited to or are heavily reliant on grayscale images [21]. As a result, only the After latent images in grayscale are currently available to be studied and used for comparison. Based on the gain in potentially available information seen here, we suggest that After images without coloring stripped away be provided, as the color may help preserve useful feature information not present in the grayscale version.

\subsection{LATENT VALUE DETERMINATION}

The subjective Latent Value Determination (see Section 4.2), was the basis for our second performance metric. Upon receiving the latent image, examiners assigned a subjective quality rating, using the 'Good, Bad, and Ugly' scale [16]. After preprocessing was applied to the 
latent image, the new version of the latent image was examined again and re-rated. Table 7 below shows the number of images assigned to each category before preprocessing, as well as the updated ratings for the preprocessed images.

Table 7: Value Determination and Re-Categorization

\begin{tabular}{|l|l|l|l|l|}
\hline Before & Good & Bad & Ugly & Total \\
\hline Good & 25 & 0 & 0 & 25 \\
\hline Bad & 23 & 12 & 0 & 35 \\
\hline Ugly & 5 & 16 & 8 & 29 \\
\hline Total & 53 & 28 & 8 & 89 \\
\hline
\end{tabular}

Across each row are the classifications for the BeforePreprocess-GrayScale image - 25 Good, $35 \mathrm{Bad}$, and 29 Ugly. Down each column are the reclassifications for the AfterPreprocessGrayScale images - 53 Good, 28 Bad, and 8 Ugly. The differences in Before/After classifications can be seen in the central cells. The 53 latent images rated as Good after preprocessing were made up of the 25 inherently Good rated images, 23 previously Bad rated images, and 5 previously Ugly rated images. The 28 latent images rated as Bad after preprocessing were made up of 12 previously Bad rated images (out of 35) and 16 previously Ugly rated images. Eight Ugly rated images remained classified as such after preprocessing. No instances of quality deterioration were found.

Table 7 shows that across quality determination categories, 49\% of latent fingerprint images were markedly improved after preprocessing. However, this percentage includes images previously rated Good. Because Good images are the highest value determination rating available and there were no instances of images going down in quality rating, we exclude these to provide a more contextually specific figure. Excluding these images, we find that $69 \%$ of Bad or Ugly images were improved by at least one quality rating after preprocessing.

\subsection{QUALITY CONFIDENCE SCORE}

The final performance metric used in our study was the Quality Confidence Score. This measurement is derived by cross referencing the position of each minutiae with the Latent Quality Map rating of where that minutiae is located. In the 'Numerical Latent Quality Map' image in Figure 9, the values corresponding to the quality mapping colors can be seen. With each number representing an 8x8 pixel area of the image, a visual representation of each quality color area can be seen. See Figure 21 for an example. 
The quality weighted score of a latent fingerprint is computed as follows: given each marked minutia in the latent image, locate its position in the quality map, and obtain its quality map value for that minutia position. The quality confidence score for the image is defined as sum of all minutia quality map values. This score is intended to measure how thoroughly a LPE could mark the features within a latent image, as well as how confident the LPE is in the definitive existence of those marked features.

After calculating the quality confidence score for each record, the mean score gain was calculated. When comparing BeforePreprocess-GrayScale images to BeforePreprocess-Color images, the mean score improved by $28 \%$. When comparing BeforePreprocess-GrayScale to AfterPreprocess-GrayScale, the mean score improved by $47 \%$. Finally, the BeforePreprocessColor to AfterPreprocess-GrayScale comparison came in at a mean score improvement of $32 \%$. Median improvement came in at 22\%, 48\%, and 42\% respectively. Using the same 89 records used for the other quality metrics, a handful were removed from the Wilcoxon Signed Ranks Test due to lack of minutiae markup and/or identical scores.

Based on the results of the Wilcoxon Signed Ranks Test as seen in Table 8, with $\alpha=.05$, the differences in quality confidence score between the BeforePreprocess-GrayScale and BeforePreprocess-Color, the BeforePreprocess-GrayScale and AfterPreprocess-GrayScale, and BeforePreprocess-Color to AfterPreprocess-GrayScale are all statistically significant.

Table 8: Wilcoxon Signed Ranks Test - Quality Confidence Score

\begin{tabular}{|l|l|l|l|}
\hline Comparison & $\mathbf{N}$ & $\mathbf{Z}$ & $\mathbf{P}$ \\
\hline BG to BC & 86 & -3.64 & $<.0003$ \\
\hline BG to AG & 87 & -4.35 & 0.000 \\
\hline BC to AG & 87 & -3.49 & $<.0005$ \\
\hline
\end{tabular}




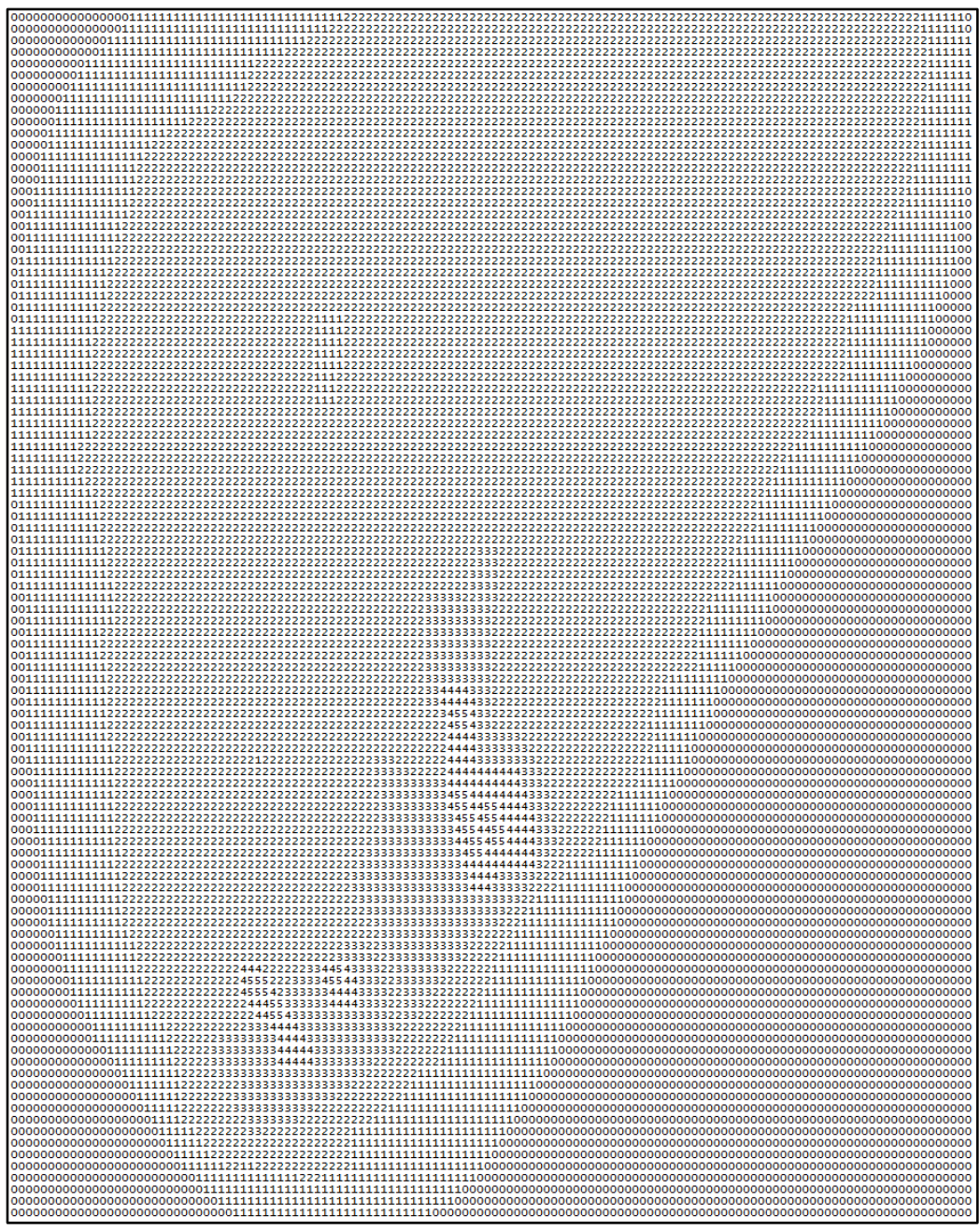

Figure 25: Quality Map Numerical Representation

\section{DISCUSSION AND FUTURE WORK}

The current forensic science landscape deems many latent fingerprint images as 'no value', leading them to be unused in any comparison process [11]. However, there may be valuable feature information still held within these latent images, revealed only once they undergo preprocessing and enhancement. Unfortunately, due to the lack of quantitative techniques for image preprocessing, many forensic laboratories do not employ or allow image preprocessing software. Though the discipline may have to wait for having latent fingerprint preprocessing as a standard operating procedure, we hope that the database we've created will serve as a foundation for future preprocessing studies. 
Based on the quantitative results of the comparison experiments described in this report, we have demonstrated that the amount of feature information held within a latent fingerprint image is significantly improved after preprocessing is applied. Though the scope of our research is limited to latent fingerprints, the design approach and analysis methods are likely applicable to other biometric comparative disciplines, including handwriting, footwear, tool marks, firearm and toolmarks, tread marks, and so on.

Future work involving the database will include another series of comparisons using the quality map, which will examine the average numerical difference between each 8x8 pixel area. Additionally, we plan to research currently available and prototype methods of computer generated feature markup to determine if they are sufficiently accurate enough for comparisons. If so, we will repeat our performance comparisons with the computer-generated markups to see if the increases in feature information remain. Finally, we will continue to provide techniques and processes enabling latent fingerprint examiners to analyze and compare evidence more effectively, as well as build foundations for future academic research and standards formulation.

\section{ACKNOWLEDGEMENTS}

The authors would like to acknowledge David L. Witzke (Foray Technologies) for his role in providing the example Before/After preprocessing comparison images, as well as Curtis Lamp (NIST) for his work in mask area calculation and visualization.

Acknowledgement is also extended to all other individuals who helped review and edit this report, ensuring that it accurately represents the hard work of all those involved.

\section{DISCLAIMER}

Any mention of commercial products or reference to commercial organizations in this report is for information only; it does not imply recommendation or endorsement by NIST nor does it imply that the products mentioned are necessarily the best available for the purpose. 


\section{REFERENCES}

[1] Melissa K. Taylor, David H. Kaye, Thomas Busey, Melissa Gische, Gerry LaPorte, Colin Aitken, Susan M. Ballou, Leonard Butt, Christophe Champod, David Charlton, Itiel E. Dror, Jules Epstein, Robert J. Garrett, Max Houck, Edward J. Imwinkelried, Ralph Keaton, Glenn Langenburg, Deborah A. Leben, Alice Maceo, Kenneth F. Martin, Jennifer L. Mnookin, Cedric Neumann, Joe Polski, Maria A. Roberts, Scott A. Shappell, Lyle Shaver, Sargur N. Srihari, Hal S. Stern, David Stoney, Anjali Swienton, Mary F. Theofanos, Robert M. Thompson, John Vanderkolk, Maria Weir, and Kasey Wertheim, "Latent Print Examination and Human Factors: Improving the Practice through a Systems Approach: The Report of the Expert Working Group on Human Factors in Latent Print Analysis.” NIST Interagency/Internal Report (NISTIR) 7842. [Online] https://www.nist.gov/publications/latent-print-examination-and-human-factorsimproving-practice-through-systems-approach. [Accessed: 25-May-2018]

[2] Edward M. Robinson, “Crime Scene Photography.” Chapter 10, Digital Imaging Technologies by David "Ski" Witzke, Third Edition, ISBN: 0128027649, ISBN-13: 9780128027646, Academic Press, Inc, Aug. 15, 2016,

[3] Jindal et. al "Exploration of latent fingerprints enhancement using soft computing techniques.” 2016 3rd International Conference on Computing for Sustainable Global Development (INDIACom), March 16-18, 2016, New Delhi, India

[4] Makhija et. al, "Performance analysis of latent fingerprint enhancement technologies.” 2017 International Conference on Innovative Mechanisms for Industry Applications (ICIMIA), 21-23 Feb. 2017, Bangalore, India

[5] JianLi, JianjiangFeng, and C.-C. JayKuo, "Deep convolutional neural network for latent fingerprint enhancement.” Signal Processing: Image Communication, Vol. 60, Feb. 2018, pg. 52-63.

[6] Haiying Guan, Paul Y. Lee, Curtis L. Lamp, Andrew M. Dienstfrey, Mary F. Theofanos, Brian C. Stanton, Matthew Schwarz, "Analysis, Comparison, and Assessment of Latent Fingerprint Image Preprocessing.” 2017 IEEE Conference on Computer Vision and Pattern Recognition Workshops (CVPRW)

[7] H. C. Lee and R. E. Gaensslen, Eds., Advances in fingerprint technology, 2nd ed. Boca Raton, Fla: CRC Press, 2001.

[8] R. Rajkumar and K. Hemachandran, "Latent Fingerprint Enhancement in Preprocessing Stage,” in International Conference on Computer Science and Intelligent Systems (CSIS '11), 2011.

[9] Kawagoe, Masahiro, and Akio Tojo. "Fingerprint Pattern Classification.” Pattern Recognition, vol. 17, no. 3, 1984, pp. 295-303. 
[10] M. Theofanos, B. Stanton, D. Witzke, and M. Schwarz, "Characterizing the Latent Fingerprint Pre-Processing Procedures,” National Institute of Standards and Technology, NIST Internal or Interagency Reports (NISTIR), 2017.

[11] B. T. Ulery, R. A. Hicklin, G. I. Kiebuzinski, M. A. Roberts, and J. Buscaglia, "Understanding the sufficiency of information for latent fingerprint value determinations," Forensic Sci. Int., vol. 230, no. 1-3, pp. 99-106, Jul. 2013.

[12] B. T. Ulery, R. A. Hicklin, M. A. Roberts, and J. Buscaglia, "Measuring What Latent Fingerprint Examiners Consider Sufficient Information for Individualization Determinations,” PLOS ONE, vol. 9, no. 11, p. e110179, Nov. 2014.

[13] P. Peterson et al., "Latent Prints: A Perspective on the State of the Science," FBI Forensic Sci. Commun., vol. 11, no. 4.

[14] "FBI Biometric Specifications (BioSpecs)." Latent Print Services, www.fbibiospecs.cjis.gov/.

[15] "The Universal Latent Workstation (ULW) Brochure” U.S. Department of Justice, Federal Bureau of Investigation (FBI), [Online] https://www.fbibiospecs.cjis.gov/Latent/PrintServices

[16] Garris, Michael D. "Fingerprint Minutiae From Latent and Matching Tenprint Images.” NIST Special Database 27, 2000

[17] “Applicant Fingerprint Form (FD-258).” FBI, FBI, 2 June 2016, www.fbi.gov/filerepository/standard-fingerprint-form-fd-258-1.pdf/view.

[18] "Recording Legible Fingerprints." FBI, FBI, 2 June 2016, www.fbi.gov/services/cjis/fingerprints-and-other-biometrics/recording-legiblefingerprints.Taylor, Melissa, et al. "Markup Instructions for Extended Friction Ridge Features.” 2013

[19] K. C. Mangold, "Data Format for the Interchange of Fingerprint, Facial \& Other Biometric Information ANSI/NIST-ITL 1-2011.” NIST Special Publication 500-290 Edition 3, 22-Aug-2016.

[20] R. Lowry, "Concepts and Applications of Inferential Statistics, by Richard Lowry," 2015. [Online]. Available: http://onlinebooks.library.upenn.edu/webbin/book/lookupid?key=olbp66608. SubChapter 12a (http://vassarstats.net/textbook/ch12a.html). [Accessed: 25-May-2018]

[21] L. M. I. Dexter and A. J. Pouratian, "Automated fingerprint identification system," US5869822 A, 09-Feb-1999. 